Pacific

Journal of

Mathematics

REPRESENTATION OF TYPES AND 3-MANIFOLDS

Luis Gerardo Valdez SÁnchez

Volume $210 \quad$ No. 2

June 2003 


\title{
REPRESENTATION OF TYPES AND 3-MANIFOLDS
}

\author{
Luis Gerardo Valdez SÁnchez
}

\begin{abstract}
According to theorems of C. Gordon, J. Luecke, and W. Parry, if a knot exterior $\boldsymbol{X}$ has two distinct planar boundary slopes $r_{1}, r_{2}$, then at least one of the manifolds $X\left(r_{1}\right), X\left(r_{2}\right)$ has a connected summand $M$ with nontrivial torsion in first homology. The 3-manifolds $M$ obtained in this way, which we call $t$-manifolds, have special Heegaard splittings, or $t$-manifold structures. In this paper we study the topology of t-manifolds from the point of view of the homology presentation matrices induced by their t-manifold structures, classify all genus two t-manifold structures, and show that, under some conditions, one of the Dehn fillings of $X$ is a connected sum of t-manifolds and (at most) one prime non t-manifold summand.
\end{abstract}

\section{Introduction.}

Let $X$ be a knot exterior, i.e., a connected, compact, orientable, irreducible 3 -manifold with torus boundary. For $r$ a slope in $\partial X$, let $X(r)$ be the manifold obtained by Dehn-filling $X$ along $r$. If $r$ and $s$ are two distinct planar boundary slopes on $\partial X$, then by $[6, \S 4]$ at least one of the manifolds $X(r), X(s)$ contains a connected summand $M$ with nontrivial torsion in first homology. Such a manifold $M$ can be constructed as follows: Let $P, Q$ be essential planar surfaces in $X$ with boundary slope $r, s$, respectively; we may assume that $P, Q$ intersect transversely in planar graphs $G_{P}=P \cap Q \subset P$ and $G_{Q}=P \cap Q \subset Q$ without boundary parallel arcs, and that any circle component of $P \cap Q$ is essential in $P$ and $Q$. By [8] and [9], $\Delta(r, s)=1$ and at least one of the two graphs, say $G_{Q}$, has a set of disk faces $\Sigma$ which represents all types. Let $\widehat{P}$ be the surface obtained by capping each boundary component of $P$ with a disk, so that $\widehat{P}=S^{2}$, and let $(E, \partial E) \subset(\widehat{P}$, int $P)$ be a disk which contains the edges of all faces in $\Sigma$. It is then possible to choose the collection $\Sigma$ so that every disk in it lies locally on the same side of $E$ (cf. $[6, \S 4])$. Subject to these constraints, we further assume that $|\Sigma|$ is as small as possible (where $|\ldots|$ stands for cardinality or number of connected components).

Now, the 2-sphere $\widehat{P}$ splits the filling solid torus of $X(r)$ into a finite collection of '1-handles' $\mathcal{H}$. Following [6, §4], let $N(E, \Sigma) \subset X(r)$ be the regular neighborhood of the set $E \cup \mathcal{H}^{*} \cup \Sigma$, where $\mathcal{H}^{*} \subset \mathcal{H}$ denotes the 
collection of 1-handles each of which is intersected by the boundary of at least one disk in $\Sigma$. Then $M$ can be taken to be the manifold $\widehat{N(E, \Sigma)}$ (where $\widehat{Y}$ is obtained by capping off each boundary 2-sphere component of $Y$ with a 3-ball); that $M$ has nontrivial torsion in first homology follows from [13]. We remark that we can take $E=\widehat{P}$ whenever $\widehat{P}$ separates, in which case $\partial N(E, \Sigma)$ consists of two 2-spheres, one of which is parallel to $\widehat{P}$ and the other having fewer than $|\partial P|$ boundary components. We call the collection $\Sigma$ a generalized Scharlemann cycle, and say that $M$ has been obtained by attaching the generalized Scharlemann cycle $\Sigma$ to $E$ (or $\widehat{P}$, as the case may be). Perhaps the simplest example of this situation is provided by the example of Gordon and Litherland [7, Appendix] (see also [15, §4]).

The construction of the manifold $M$ can be abstracted as follows: Throughout, we work in the PL-category, and all manifolds are assumed to be compact and orientable. Some familiarity of the reader with the papers $[\mathbf{4}$, Chapter 2] and $[\mathbf{8}, \mathbf{6}]$ is assumed; we also refer the reader to $[\mathbf{3}]$ for standard definitions and notation about Heegaard splittings. Let $H_{n}$ be a genus $n$ handlebody with a fixed complete disk system $\boldsymbol{D}=\left\{D_{1}, \ldots, D_{n}\right\}$; we call the circles $\partial \boldsymbol{D}=\left\{\partial D_{1}, \ldots, \partial D_{n}\right\}$ the meridians of $H_{n}$. A collection of disjoint circles $\boldsymbol{C}$ embedded in $\partial H_{n}$ is said to intersect $\partial \boldsymbol{D}$ coherently if, for each $c \in \boldsymbol{C}$ and $D \in \boldsymbol{D}$, the intersection $c \cap \partial D$ is transverse and $|c \cdot D|=|c \cap D|$. This last condition can be restated as saying that each curve in $\boldsymbol{C}$ intersects a given meridian circle of $H_{n}$ always in the same direction.

Now fix orientations of $H_{n}$ and the components of $\partial \boldsymbol{D}$ and $\boldsymbol{C}$. For each circle $c$ in $\boldsymbol{C}$, let $a(c)=\left(a(c)_{1}, \ldots, a(c)_{n}\right)$ be the ordered $n$-tuple whose $i$-th entry is given by the algebraic intersection number $c \cdot D_{i}$ of $c$ with $\partial D_{i}$. The collection $\boldsymbol{C}$ represents all types (relative to the meridians of $H_{n}$ ) if the set of integral vectors $\{a(c), c \in C\}$ represents all $n$-types in the sense of Parry [13], that is, if for any real vector $x=\left(x_{1}, \ldots, x_{n}\right) \in \mathbb{R}^{n}$ there is a vector $a(c) \in \boldsymbol{C}$ such that all the nonzero terms in the expansion of the standard inner product $\langle a(c), x\rangle$ have the same sign.

For any collection $\boldsymbol{C}$ of disjoint circles embedded in $\partial H_{n}$ and satisfying the conditions:

1) $\boldsymbol{C}$ intersects $\partial \boldsymbol{D}$ coherently,

2) $C$ represents all types,

3) no proper subcollection of $\boldsymbol{C}$ represents all types, and

4) every meridian circle in $\partial \boldsymbol{D}$ is intersected nontrivially by at least one circle in $\boldsymbol{C}$,

the 3 -manifold $\mathcal{M}$ obtained by attaching 2 -handles to $\partial H_{n}$ along the circles in $\boldsymbol{C}$ and capping off any resulting 2 -sphere boundary components will be called a $t$-manifold. The particular data $\boldsymbol{S}=\left(H_{n}, \boldsymbol{D}, \boldsymbol{C}\right)$ used to construct $\mathcal{M}$ will be called a genus $n$ t-manifold structure of $\mathcal{M}$, and we will refer to 
the curves in $\boldsymbol{C}$ as the attaching circles of the t-manifold structure. Observe that the integral matrix $A_{\mathcal{M}}$ whose rows are the vectors $\{a(c), c \in C\}$ is a presentation matrix for $H_{1}(\mathcal{M})$.

It follows from the definition that any genus one t-manifold is either $S^{3}$ or a lens space. In the first part of this paper we give a complete classification of the 3-manifolds that admit a genus two t-manifold structure; this is the content of our first theorem:

Theorem 1.1. If $\mathcal{M}$ is a t-manifold with a genus two t-manifold structure, then $\mathcal{M}=M_{1} \cup_{\partial} M_{2}$, where each $M_{i}$ is a Seifert fibered space over a disk with at most two singular fibers, such that the regular fibers of $M_{1}, M_{2}$ intersect in one point in $\partial M_{1}=\partial M_{2}$.

In particular, $\mathcal{M}$ is either toroidal, a Seifert fibered space over a 2 -sphere with 3 singular fibers, a lens space, or $S^{3}$, and it is irreducible but not always Haken.

It is possible to determine when a 3 -manifold $\mathcal{M}$ admitting a decomposition of the form $\mathcal{M}=M_{1} \cup_{\partial} M_{2}$ as in Theorem 1.1 is indeed a t-manifold, via coherency invariants which are derived from the Euler numbers of $M_{1}$ and $M_{2}$. These invariants are constructed via a function $\llbracket \ldots \rrbracket: \mathbb{R} \rightarrow \frac{1}{2} \mathbb{Z}$ defined as follows: $\llbracket x \rrbracket=[x]+1 / 2$ for each real number $x$ that is not an integer (here $[\ldots]$ denotes the greatest integer function), and $\llbracket x \rrbracket=x$ for each integer $x$. Observe that $\llbracket . . \rrbracket$ is an odd periodic function of period 1.

\section{Theorem 1.2.}

(a) Let $\mathcal{M}$ be a 3-manifold of the form $\mathcal{M}=M \cup_{\partial} M^{\prime}$, where $M, M^{\prime}$ are Seifert-fibered spaces over a disk with two singular fibers, glued along their torus boundaries in such a way that their regular fibers intersect in one point. Let the Seifert invariants of $M$ and $M^{\prime}$ be $\left(a_{1}, p_{1} ; a_{2}, p_{2}\right)$ and $\left(a_{1}^{\prime}, p_{1}^{\prime} ; a_{2}^{\prime}, p_{2}^{\prime}\right)$, where $a_{i}, a_{i}^{\prime}>1$ for each $i$. Then $\mathcal{M}$ has a genus two t-manifold structure iff $\left(\llbracket p_{1} / a_{1} \rrbracket+\llbracket p_{2} / a_{2} \rrbracket\right) \cdot\left(\llbracket p_{1}^{\prime} / a_{1}^{\prime} \rrbracket+\llbracket p_{2}^{\prime} / a_{2}^{\prime} \rrbracket\right)<0$.

(b) Let $\mathcal{M}$ be a Seifert fibered manifold over the 2-sphere with three singular fibers and Seifert invariant $\left(a_{1}, p_{1} ; a_{2}, p_{2} ; a_{3}, p_{3}\right)$, where $a_{i}>$ 1 for each $i$. Then $\mathcal{M}$ admits a genus two t-manifold structure iff $\llbracket p_{1} / a_{1} \rrbracket+\llbracket p_{2} / a_{2} \rrbracket+\llbracket p_{3} / a_{3} \rrbracket \neq \pm 1 / 2$.

Before discussing higher genus t-manifold structures, we slightly generalize the notion of types representation. We will say that a nonempty set of vectors $X$ in $\mathbb{R}^{n}$ represents all types if for each vector $v \in \mathbb{R}^{n}$ there is a vector $x \in X$ such that all the nonzero terms in the expansion of the standard inner product $\langle v, x\rangle$ have the same sign. Observe that we allow sets of nonintegral vectors to represent all types. We extend this definition to matrices, and say that a real $k \times n$ matrix represents all types if its set of row vectors, viewed as elements of $\mathbb{R}^{n}$, represent all types. The following result is the starting point for our analysis of the topological structure of 
t-manifolds; it essentially follows from Lemma 4.4 in [9] (see also [6, Lemma $3.2]$ ), though our approach to the proof is somewhat different:

Theorem 1.3. Let $A$ be a $k \times n$ matrix representing all types. Then one of the following must hold:

(a) A has a column of zeroes;

(b) A proper subset of the rows of A represents all types;

(c) A has rank $n$.

Matrices which represent all types and do not satisfy conditions (a) or (b) of Theorem 1.3 will be called proper matrices. In this sense, a finite set of vectors in $\mathbb{R}^{n}$ which represents all types 'efficiently' must be a spanning set, and proper matrices are those matrices which represent all types 'efficiently'. Observe that, for a t-manifold $M$ with t-manifold structure $\boldsymbol{S}=\left(H_{n}, \boldsymbol{D}, \boldsymbol{C}\right)$, the associated integral matrix $A_{M}$ is proper. The following result summarizes some immediate properties of $M$ and the matrix $A_{M}$ (cf. [6, Theorem 4.3]):

Theorem 1.4. Let $M$ be any $t$-manifold with a genus $n$-manifold structure and $t$-presentation matrix $A_{M} \neq(1)$. Then:

(a) $A_{M}$ has rank $n$;

(b) $M$ is closed;

(c) $H_{1}(M)$ is finite and nontrivial.

It is possible to extract some more information about the topology of a t-manifold by studying the distribution of zero entries in its t-presentation matrices. Define the girth of any $k \times l$ matrix as the number $k+l$. The zero girth (0-girth for short) of a matrix is then defined as the largest girth of any of its zero submatrices. The following result gives a bound on the 0-girth of a square matrix that represents all types properly:

Theorem 1.5. An $n \times n$ matrix $A$ that represents all types is proper iff its 0 -girth is at most $n-1$.

This result can be used to give a nice characterization of proper square matrices (see Corollary 5.7) and, along with well-known results of A. Casson and $\mathrm{C}$. Gordon on reducible Heegaard splittings of 3-manifolds, to show that some t-manifolds having 'square' t-manifold structures of smallest possible genus may not be hyperbolic.

Theorem 1.6. Let $M$ be a t-manifold with a genus $n$-manifold structure and square t-presentation matrix $A_{M}$. If the Heegaard genus of $M$ is $n$, then either:

(a) $M$ is reducible or contains an incompressible embedded torus, or

(b) the 0-girth of $A_{M}$ is at most $n-2$. 
Moreover, if the matrix $A_{M}$ has a $2 \times 2$ submatrix which does not represent all types, then (a) holds.

A t-manifold can be thought of as a generalized lens space, just like a generalized Scharlemann cycle generalizes the notion of Scharlemann cycle. From this point of view, it may not be surprising that no t-manifold with a Heegaard genus two t-manifold structure is hyperbolic. One can then ask whether there are hyperbolic t-manifolds at all. The above theorem gives a partial answer to this question in the negative, but the general case is still open.

We also prove that t-manifolds arise in a natural way from Dehn fillings of knot spaces along different planar boundary slopes; the following result is a mild extension of [15, Theorem 1.3]:

Theorem 1.7. Let $X$ denote a knot space with distinct planar boundary slopes $r, s$. Then, either $X(s)$ has a lens space ( $t$-manifold, resp.) connected summand or $X(r)$ has a t-manifold (lens space, resp.) connected summand and at most one prime factor of $X(r)$ is not part of some $t$-manifold (it is not a lens space, resp.) connected summand.

The paper is organized as follows: We prove in Section 2 some basic results on systems of arcs in 4-punctured 2-spheres, which will be used in Section 3 to prove Theorem 1.1. Theorem 1.2 is proved in Section 4, where coherency invariants of the Seifert fiber structures are introduced. Theorem 1.3 and Theorem 1.5 are proved in Section 5, along with other properties of proper matrices and their 0-girths. Proofs of Theorems 1.4, 1.6, and 1.7 are given in Section 6.

I want to thank Andrew Casson for many suggestions and helpful discussions on these results, some of which formed part of my thesis.

\section{Systems of arcs in 4-punctured 2-spheres.}

Let $H$ be an orientable genus two handlebody with fixed meridian disks $D, \bar{D}$ and corresponding meridian circles $m=\partial D, \bar{m}=\partial \bar{D}$, and let $\gamma$ be an essential closed 1-submanifold of $\partial H$ that intersects $m \cup \bar{m}$ transversely. Cutting $\partial H$ along the meridian circles $m, \bar{m}$ yields a 4 -punctured sphere $S_{0}$; denote the punctures corresponding to $m$ by $m_{1}, m_{2}$, and those corresponding to $\bar{m}$ by $\bar{m}_{1}, \bar{m}_{2}$. We say that the collection $\Gamma(\gamma)=\gamma \cap S_{0} \subset S_{0}$ is standard if its components are essential arcs in $S_{0}$ which can be sorted out into six subcollections $\Gamma_{i}(\gamma), 1 \leq i \leq 6$, as follows:

$\Gamma_{1}(\gamma)$ : Arcs connecting $m_{1}$ and $\bar{m}_{1}, \quad \Gamma_{2}(\gamma)$ : Arcs connecting $m_{2}$ and $\bar{m}_{2}$, $\Gamma_{3}(\gamma)$ : Arcs connecting $m_{1}$ and $\bar{m}_{2}, \quad \Gamma_{4}(\gamma)$ : Arcs connecting $m_{2}$ and $\bar{m}_{1}$, $\Gamma_{5}(\gamma)$ : Arcs connecting $m_{1}$ and $m_{2}, \quad \Gamma_{6}(\gamma)$ : Arcs connecting $\bar{m}_{1}$ and $\bar{m}_{2}$.

Observe that $\Gamma(\gamma)$ does not contain any arc connecting a boundary component of $S_{0}$ to itself (see Figure 1) and has no circle components. We will 


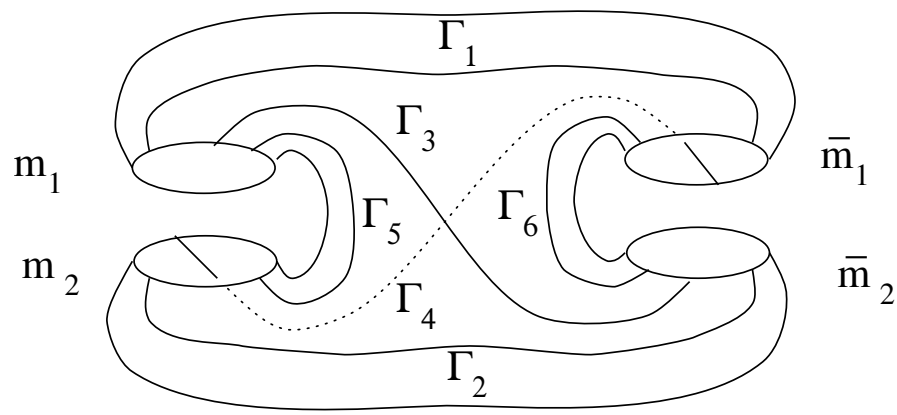

$\mathrm{S}_{0}$

Figure 1. $\Gamma(\gamma) \subset S_{0}$.

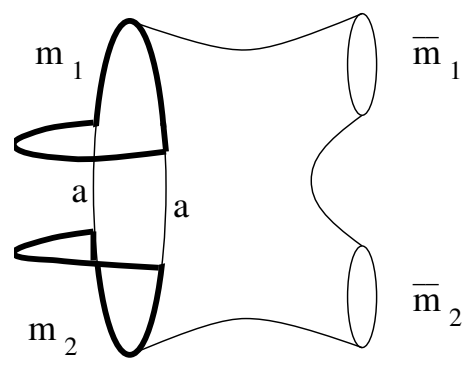

(a)

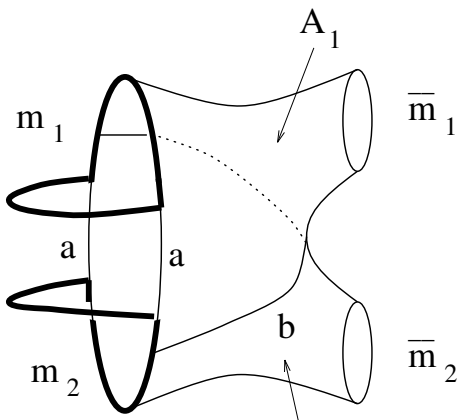

$\mathrm{A}_{2}$

(b)

Figure 2. The four-punctured sphere $S_{0}$ cut along the arc $a$.

omit the argument $\gamma$ when this does not cause any confusion. Two $\operatorname{arcs} a, b$ of $\Gamma(\gamma)$ are parallel in $S_{0}$ if the closure of one of the components of $S_{0} \backslash(a \cup b)$ is a rectangle.

Lemma 2.1. Let $\Gamma(\gamma)$ be a standard system of arcs in $S_{0}$.

(a) If $\Gamma_{5} \neq \emptyset\left(\Gamma_{6} \neq \emptyset\right.$, resp. $)$, then either:

(i) Each collection $\Gamma_{5}$ and $\Gamma_{6}$ consists of mutually parallel arcs, or

(ii) $\Gamma_{5}\left(\Gamma_{6}\right.$, resp.) contains two non-parallel arcs $a, b$ such that every arc in $\Gamma_{5}\left(\Gamma_{6}\right.$, resp. $)$ is parallel to one of a or $b$, and $\Gamma_{6}\left(\Gamma_{5}\right.$, resp. $)$ is empty.

(b) Let $n_{i}=\left|\Gamma_{i}\right|$ for each $i$. Then $n_{1}=n_{2}$ and $n_{3}=n_{4}$.

Proof. Suppose that $\Gamma_{5}$ contains two non-parallel $\operatorname{arcs} a, b$. Cut $S_{0}$ along $a$ to obtain a 3 -punctured sphere $S_{0}^{\prime}$ with boundary components $\bar{m}_{1}, \bar{m}_{2}, C$ and 
containing $b$, where $C$ consists of one arc from each of $m_{1}$ and $m_{2}$ connected by two arcs parallel to $a$, as indicated in Figure 2(a).

If there is an arc $c$ in $S_{0}^{\prime}$ disjoint from $b$ connecting $\bar{m}_{1}$ and $\bar{m}_{2}$, then cutting $S_{0}^{\prime}$ along $c$ yields an annulus $A$ with two boundary components $C, C^{\prime}$ and containing $b$. Since both endpoints of $b$ lie on $C, b$ must be boundary-parallel to $C$ in $A$ and hence it must be parallel to $a$ in $S_{0}$, which is a contradiction. Therefore, $\Gamma_{6}$ is empty and $b$ separates $\bar{m}_{1}$ from $\bar{m}_{2}$ in $S_{0}^{\prime}$ (Figure 2(b)).

Cut $S_{0}^{\prime}$ along $b$ to obtain two annuli $A_{1}, A_{2}$, each containing $\bar{m}_{1}, \bar{m}_{2}$, respectively. Then any arc in $A_{1}$ or $A_{2}$ connecting $m_{1}$ and $m_{2}$ must be boundary-parallel (see Figure 2(b)), hence parallel in $S_{0}$ to one of $a$ or $b$.

Suppose now that all the arcs of $\Gamma_{5}$ are parallel. By amalgamating all these arcs if necessary, we may assume that $\Gamma_{5}$ contains only one arc $d$. Since $d$ does not separate $\bar{m}_{1}$ from $\bar{m}_{2}, \Gamma_{6}$ may not be empty. If indeed $\Gamma_{6}$ is not empty, then all its arcs must be mutually parallel, for otherwise the argument above would apply to show that $\Gamma_{5}$, contrary to hypothesis, is empty. This establishes Part (a).

For Part (b), observe that the number of endpoints of $\operatorname{arcs}$ of $\Gamma$ in $m_{1}$ and $m_{2}$ match, and so

$$
n_{1}+n_{3}+n_{5}=n_{2}+n_{4}+n_{5},
$$

which implies that $n_{1}+n_{3}=n_{2}+n_{4}$. Similarly, comparing the number of endpoints of $\Gamma$ in $\bar{m}_{1}$ and $\bar{m}_{2}$ yields $n_{1}+n_{4}=n_{2}+n_{3}$, whence $n_{1}=n_{2}$ and $n_{3}=n_{4}$.

We call a standard system of $\operatorname{arcs} \Gamma(\gamma) \subset S_{0}$ split whenever the arcs in $\Gamma_{5}(\gamma)$ or $\Gamma_{6}(\gamma)$ split into two nonempty, non-parallel collections of parallel arcs as in Lemma 2.1 (a)(ii). Otherwise, $\Gamma(\gamma)$ is said to be non-split.

A waist circle of $\partial H$ is an essential circle $w \subset \partial H$ separating $m$ from $\bar{m}$; such a circle always bounds a disk properly embedded in $H$. Any waist circle is said to be $\Gamma(\gamma)$-simple if it is transverse to $\Gamma(\gamma)$ and disjoint from $\Gamma_{5}(\gamma) \cup \Gamma_{6}(\gamma)$.

Lemma 2.2. There exists a $\Gamma$-simple waist circle $w$ iff $\Gamma$ is non-split. Such a waist circle is unique up to isotopy if $\Gamma_{5} \cup \Gamma_{6}$ is not empty, and may be assumed to intersect each arc of $\Gamma \backslash\left(\Gamma_{5} \cup \Gamma_{6}\right)$ in one point.

Proof. Let $w$ be a $\Gamma$-simple waist circle. Without loss of generality, we may assume $\Gamma_{5}$ is not empty. If $\Gamma_{5}$ contains two $\operatorname{arcs} a, a^{\prime}$, then the component of $S_{0}$ cut along $w \cup a$ which contains $a^{\prime}$ is an annulus, with one boundary component consisting of one arc from each of the circles $m_{1}, m_{2}$ connected by two arcs parallel to $a$, while the other boundary component is a curve parallel to $w$. It is then clear that $a^{\prime}$ must be parallel to $a$, so $\Gamma$ is non-split.

Conversely, suppose $\Gamma$ is non-split. Without loss of generality, we may assume that $\Gamma_{5}$ contains exactly one arc (either by creating it or by amalgamating all its arcs). Denote such an arc by $b$, and let $N$ be a regular 
neighborhood of $b$ in $S_{0}$, small enough to be disjoint from all other arcs in $\Gamma$. We then obtain a $\Gamma$-simple waist circle $w$ by band-connecting $m_{1}$ and $m_{2}$ along $N$. Observe that each arc of $\Gamma \backslash\left(\Gamma_{5} \cup \Gamma_{6}\right)$ is intersected by $w$ exactly in one point.

Now suppose $w$ is a $\Gamma$-simple waist in $S_{0}$ and that $\Gamma_{5}$ contains an $\operatorname{arc} c$. Cut $S_{0}$ along the arc $c$ to obtain a 3-punctured sphere $S_{0}^{\prime}$ with boundary components $\bar{m}_{1}, \bar{m}_{2}, C$, where $C$ consists of pieces from $m_{1}, m_{2}$ and two arcs parallel to $c$. Since $w$ separates $\bar{m}_{1} \cup \bar{m}_{2}$ from $C, w$ and $C$ cobound an annulus in $S_{0}^{\prime}$ and are therefore parallel in $S_{0}$. The uniqueness of $w$ up to isotopy follows.

\section{Genus two t-manifold structures.}

This section is devoted to the proof of Theorem 1.1. The properties of standard systems of arcs established in the previous section will enable us to get a detailed picture of genus two t-manifold structures.

Clearly, a genus two t-manifold structure on a 3-manifold $\mathcal{M}$ has exactly two attaching curves, and since its first homology is finite, $\mathcal{M}$ is a closed manifold. Denote the meridian circles of the genus two handlebody $H$ by $u, v$, and assume that $\mathcal{M}$ has a t-manifold structure consisting of two attaching circles $x, y$ embedded in $\partial H$. Hence $H^{\prime}=\overline{M \backslash H}$ is a handlebody and so the pair $\left(H, H^{\prime}\right)$ is a Heegaard splitting of $\mathcal{M}$. Observe that the circles $u, v$ in $\partial H^{\prime}$ also give a t-manifold structure to $\mathcal{M}$ with respect to the meridians $x, y$ of $H^{\prime}$.

We denote $\partial H$ cut along $u \cup v$ by $S_{0}$, and label its boundary components by $u_{1}, u_{2}$ and $v_{1}, v_{2}$. Similarly, we denote $\partial H^{\prime}$ cut along $x \cup y$ by $S_{0}^{\prime}$ and label its boundary components by $x_{1}, x_{2}$ and $y_{1}, y_{2}$.

\section{Lemma 3.1.}

(a) The collections of arcs $\Gamma(x \cup y) \subset S_{0}$ and $\Gamma(u \cup v) \subset S_{0}^{\prime}$ are standard.

(b) Without loss of generality, we may assume that

$$
\begin{array}{lll}
\Gamma_{1}(x \cup y) \cup \Gamma_{2}(x \cup y) \subset x & \text { and } & \Gamma_{3}(x \cup y) \cup \Gamma_{4}(x \cup y) \subset y, \\
\Gamma_{1}(u \cup v) \cup \Gamma_{2}(u \cup v) \subset u & \text { and } & \Gamma_{3}(u \cup v) \cup \Gamma_{4}(u \cup v) \subset v .
\end{array}
$$

(c) Each of the collections $\Gamma_{i}(x \cup y), \Gamma_{i}(u \cup v)$ is nonempty for $1 \leq i \leq 4$ and consists of parallel arcs.

Proof. From the definition of t-manifold, no arc in $\Gamma(x \cup y)$ connects a boundary component of $S_{0}$ to itself; hence $\Gamma(x \cup y)$ is standard, so (a) follows.

Suppose now that an arc of $\Gamma_{1}(x \cup y)$ is part of $x$, oriented to run from $u_{1}$ to $v_{1}$. Then $x$ must intersect $u$ always from $u_{2}$ to $u_{1}$, and $v$ from $v_{1}$ to $v_{2}$. By coherency and the fact that $x$ and $y$ must represent all types, it follows that $\Gamma_{1}(x \cup y) \cup \Gamma_{2}(x \cup y) \subset x$ and $\Gamma_{3}(x \cup y) \cup \Gamma_{4}(x \cup y) \subset y$. The other cases are similar, so (b) holds. 


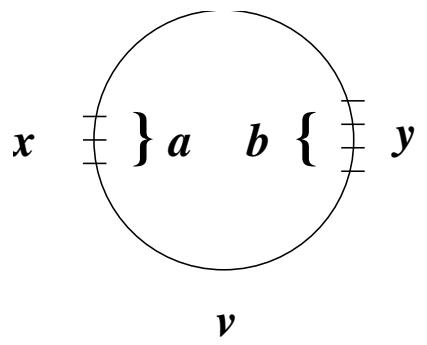

Figure 3.

If, say, $\Gamma_{1}(x \cup y)$ is empty, then $x$ must be disjoint from one of $u$ or $v$, violating condition 4) in the definition of t-manifold. The fact that the collections in (c) consist of parallel arcs follows now from Lemma 2.1.

Let $n_{i}=\left|\Gamma_{i}(x \cup y)\right|$ and $n_{i}^{\prime}=\left|\Gamma_{i}(u \cup v)\right|$. From Lemma 2.1(b), $n_{i}=n_{j}$ and $n_{i}^{\prime}=n_{j}^{\prime}$ for $\{i, j\}=\{1,2\},\{3,4\}$. For convenience, we will use the notation:

$$
a=n_{1}, \quad b=n_{3}, \quad \alpha=n_{1}^{\prime}, \quad \beta=n_{3}^{\prime} .
$$

The proof of Theorem 1.1 now splits into two cases:

Case 1. Both $\Gamma(x \cup y) \subset S_{0}$ and $\Gamma(u \cup v) \subset S_{0}^{\prime}$ are split.

Case 2. Either $\Gamma(x \cup y) \subset S_{0}$ or $\Gamma(u \cup v) \subset S_{0}^{\prime}$ is non-split.

Proof of Case 1. Suppose that $\Gamma_{5}(x \cup y)$ splits, so that $\Gamma_{6}(x \cup y)$ is empty by Lemma 2.1(a)(ii). Hence, the endpoints of $\Gamma(x \cup y)$ in $v_{1}$ are just those of $\Gamma_{1}(x \cup y)$ and $\Gamma_{4}(x \cup y)$. Since $\Gamma_{1}(x \cup y) \subset x$ and $\Gamma_{4}(x \cup y) \subset y, x \cup y$ must intersect $v$ in the pattern of Figure 3. Therefore, each of $\Gamma_{3}(u \cup v)$ and $\Gamma_{4}(u \cup v)$ must consist of exactly one arc, so $\beta=1$. If now, say, $\Gamma_{5}(u \cup v) \subset S_{0}^{\prime}$ splits, so $\Gamma_{6}(u \cup v)$ is empty by Lemma 2.1(a)(ii), then

$$
|v \cap y|=\left|v_{1} \cap y\right|=\left|\Gamma_{6}(u \cup v) \cap v\right|+\left|\Gamma_{4}(u \cup v)\right|=1
$$

and so $v$ and $y$ intersect in one point. It follows that the Heegaard splitting $\left(H, H^{\prime}\right)$ of $\mathcal{M}$ can be reduced to a genus one Heegaard splitting; hence $\mathcal{M}$ is a lens space and has the form required in the theorem.

Proof of Case 2. Suppose that $\Gamma(x \cup y)$ is non-split. Then Lemma 2.2 guarantees the existence of a $\Gamma(x \cup y)$-simple waist circle $w$ of $H$. We proceed according to two subcases:

Subcase 2(a). Either $a=1$ or $b=1$.

Assume, without loss of generality, that $a=1$. Let $D_{w} \subset H, D_{x} \subset H^{\prime}$ be properly embedded disks bounded by $w$ and $x$, respectively. By construction (see Lemma 2.2), $w$ intersects each arc of $\Gamma_{1}(x \cup y) \cup \Gamma_{2}(x \cup y) \subset x$ in one point and hence (since $\left.a=n_{1}=n_{2}=1\right) x$ in two points. 


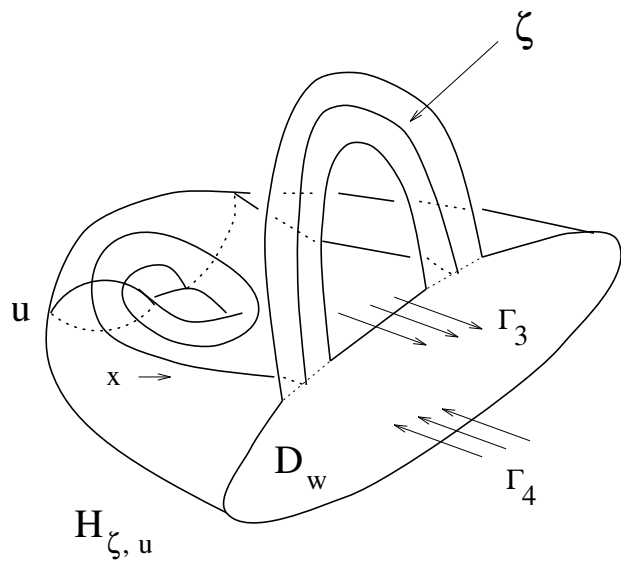

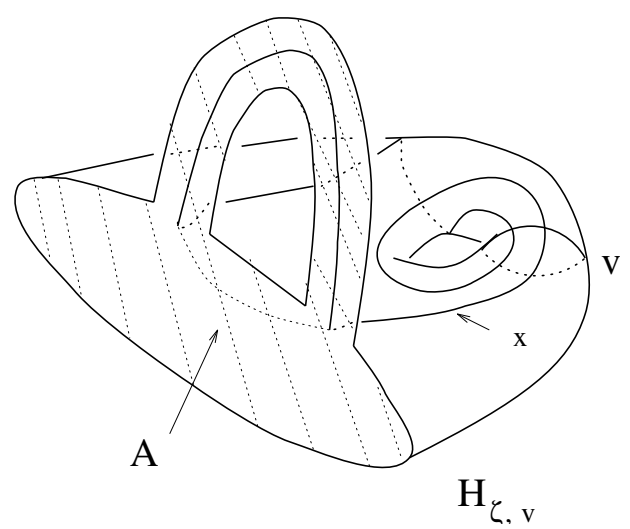

Figure 4.

Let $\zeta$ be a properly embedded arc in $D_{x}$ connecting these two points, and let $N_{\zeta}$ be a small regular neighborhood of $\zeta$ in $H^{\prime}$. The genus three handlebody $H_{\zeta}=H \cup N_{\zeta}$ contains a properly embedded annulus $A_{\zeta}=$ $D_{w} \cup B$, where $B$ is a properly embedded band in $N_{\zeta}$, that separates $H_{\zeta}$ into a pair of genus two handlebodies $H_{\zeta, u}, H_{\zeta, v}$ containing $u, v$, respectively. Observe that $\zeta$ can be extended to a core of the annulus $A_{\zeta}$ via an arc in $D_{w}$ (see Figure 4).

Now, $N_{\zeta}$ separates $D_{x}$ into two disks $D_{x, u}$ and $D_{x, v}$, properly embedded in $H_{\zeta, u}$ and $H_{\zeta, v}$, respectively; since these two disks intersect the meridians of the $N_{\zeta}$-handles of $H_{\zeta, u}, H_{\zeta, v}$ in one point, respectively, it follows that

$$
H \cup N\left(D_{x}\right)=\left(H_{\zeta, u} \cup N\left(D_{x, u}\right)\right) \cup_{A_{\zeta}}\left(H_{\zeta, v} \cup N\left(D_{x, v}\right)\right)=V_{\zeta, u} \cup_{A_{\zeta}} V_{\zeta, v},
$$

where $V_{\zeta, u}, V_{\zeta, v}$ are solid tori whose meridian circles intersect the core of $A_{\zeta}$ in $s=|x \cap u|$ and $t=|x \cap v|$ points, respectively. Hence, $N\left(H \cup D_{x}\right)$ is a Seifert fibered space over a disk with (at most) two singular fibers of indices $s$ and $t$.

It only remains to attach a 2-handle to $H \cup N\left(D_{x}\right)$ along $y$ and cap off the resulting sphere component to obtain $\mathcal{M}$. Observe that $y$ intersects each boundary component of $A_{\zeta}$ in $b$ points (recall $b=n_{3}=n_{4}$, see Figure 4). Therefore, $\mathcal{M}$ is a Seifert fibered space over the 2 -sphere with (at most) three singular fibers of indices $s, t$ and $b$. Replacing the fibration in a fibered solid torus neighborhood of some singular fiber in a suitable way shows that $\mathcal{M}$ has the required form.

Subcase 2(b). $a>1$ and $b>1$. 


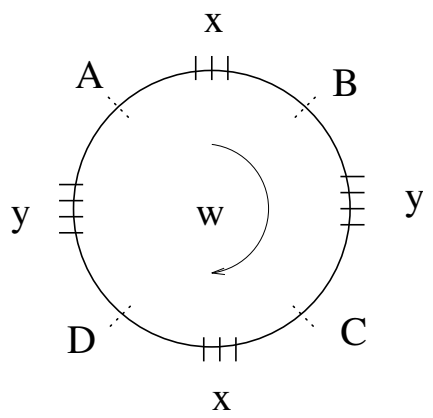

Figure 5.

If $\Gamma(u \cup v) \subset S_{0}^{\prime}$ splits then $a=1$ or $b=1$ according to the first half of the argument for Case 1 above. Therefore $\Gamma(u \cup v)$ is non-split; by Subcase 2(a), we may also assume that $\alpha>1$ and $\beta>1$.

By construction (see Lemma 2.2), $w$ intersects $x \cup y$ in the pattern given in Figure 5, from which we deduce that:

(1) $\Gamma(w) \subset S_{0}^{\prime}$ is standard: For no arc of $\Gamma(w)$ in $S_{0}^{\prime}$ runs from a component of $\partial S_{0}^{\prime}$ to itself.

(2) $\left|\Gamma_{i}(w)\right|=1$ for $1 \leq i \leq 4$.

(3) $|w \cap x|=2+\left|\Gamma_{5}(w)\right|$ and $|w \cap y|=2+\left|\Gamma_{6}(w)\right|$ : To see this, split $w$ at the four points $A, B, C, D$ marked in Figure 5. The arc $A B$ contains a subset $\Gamma_{5}^{\prime}(w)$ of the arcs in $\Gamma_{5}(w)$, while the arc $C D$ contains the remaining arcs $\Gamma_{5}^{\prime \prime}(w)$. Hence, $|A B \cap x|=1+\left|\Gamma_{5}^{\prime}(w)\right|$ and $|C D \cap x|=$ $1+\left|\Gamma_{5}^{\prime \prime}(w)\right|$, so that $|w \cap x|=2+\left|\Gamma_{5}(w)\right|$ holds. The other equality follows in a similar way.

(4) $|w \cap x|=2 a$ and $|w \cap y|=2 b$ : This follows from the construction of $w$ in Lemma 2.2.

(5) $\Gamma(w) \subset S_{0}^{\prime}$ is non-split: For suppose that $\Gamma_{5}(w) \subset S_{0}^{\prime}$ splits, so that $\Gamma_{6}(w)$ is empty. Comparing items (3) and (4) above yields $2=2 b$, hence $b=1$, contrary to hypothesis.

These facts imply that $\Gamma(u \cup v \cup w) \subset S_{0}^{\prime}$, which is automatically standard, is also non-split; a similar remark holds for the collection $\Gamma\left(x \cup y \cup w^{\prime}\right) \subset$ $S_{0}$. Let $w^{\prime}$ be a $\Gamma(u \cup v \cup w)$-simple waist circle which bounds a properly embedded disk $D_{w^{\prime}}$ in $H^{\prime}$.

Claim 1. $\left|w \cap w^{\prime}\right|=4$ and $w$ is a $\Gamma\left(x \cup y \cup w^{\prime}\right)$-simple waist.

Proof. Since $w^{\prime}$ is a $\Gamma(w)$-simple waist of $H^{\prime}$, then $w^{\prime}$ intersects $w$ only at the $\operatorname{arcs} \Gamma_{i}(w)$ for $i=1, \ldots, 4$. By item (2) above, we know that $\left|\Gamma_{i}(w)\right|=1$ for $i=1, \ldots, 4$. Hence,

$$
\left|w \cap w^{\prime}\right|=\left|\Gamma_{1}(w)\right|+\left|\Gamma_{2}(w)\right|+\left|\Gamma_{3}(w)\right|+\left|\Gamma_{4}(w)\right|=4 .
$$

In particular, this implies that $w$ is a $\Gamma\left(x \cup y \cup w^{\prime}\right)$-simple waist. 


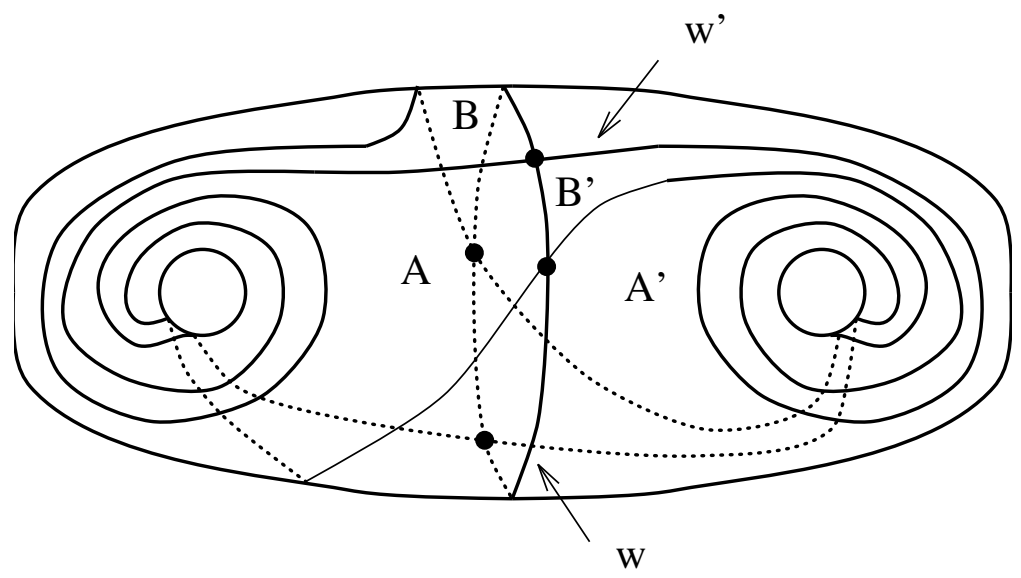

Figure 6. The waist circles $w$ and $w^{\prime}$ in $\partial H$.

Claim 2. The circles $w \cup w^{\prime}$ split the surface $S=\partial H=\partial H^{\prime}$ into two bands (rectangles) $B, B^{\prime}$ and two annuli $A, A^{\prime}$.

Proof. Each of the circles $w, w^{\prime}$ separates $S$ into two once-punctured tori. Let $T_{w}$ be the closure of one of the components of $S \backslash w$, so that $T_{w}$ is a punctured torus with boundary $w$. Since $w^{\prime}$ separates $S$, the two $\operatorname{arcs}$ of $w^{\prime} \cap T_{w}$ must be parallel in $T_{w}$ and therefore separate $T_{w}$ into a band $B$ and an annulus $A$. Similarly, $w^{\prime}$ separates the other component of $S \backslash w$ into a band $B^{\prime}$ and an annulus $A^{\prime}$ (see Figure 6).

The disk $D_{w}$ separates $H$ into two components whose closures are solid tori $V_{u}, V_{v}$ with meridian circles $u \subset \partial V_{u}$ and $v \subset \partial V_{v}$. Without loss of generality, we may assume that $\partial V_{u}=A \cup B \cup D_{w}$ and $\partial V_{v}=A^{\prime} \cup B^{\prime} \cup$ $D_{w}$. Similarly, the waist disk $D_{w^{\prime}}$ separates $H^{\prime}$ into two components whose closures are solid tori $V_{x}, V_{y}$, with meridian circles $x \subset \partial V_{x}$ and $y \subset \partial V_{y}$. Here, we may assume that $\partial V_{x}=A \cup B \cup D_{w^{\prime}}$ and $\partial V_{y}=A^{\prime} \cup B^{\prime} \cup D_{w^{\prime}}$.

Now let $T$ be the torus $D_{w} \cup B \cup B^{\prime} \cup D_{w^{\prime}}$, which is embedded in $\mathcal{M}$. It follows from the previous paragraph (see also Figure 6) that $T$ separates $\mathcal{M}$ into two components whose closures are $V_{u} \cup_{A} V_{x}$ and $V_{v} \cup_{A^{\prime}} V_{y}$. Since each arc of $w$ in $\partial V_{x}$ intersects $x$ geometrically in $a$ points (see Figure 5), the core of $A$ must intersect $x$ geometrically in $a$ points. Similarly, the core of $A$ intersects $u$ geometrically in $\alpha$ points, while the core of $A^{\prime}$ intersects $y$ and $v$ geometrically in $b$ and $\beta$ points, respectively.

The fibration of $A$ by core circles can be easily extended to a Seifert fibration of $V_{u}$ and $V_{x}$, with singular fibers of indices $a$ and $\alpha$, respectively, while the circle fibration of $A^{\prime}$ extends to a Seifert fibration of $V_{v}$ and $V_{y}$ with singular fibers of indices $b$ and $\beta$, respectively. Hence, the manifolds 
$M_{1}=V_{u} \cup_{A} V_{x}$ and $M_{2}=V_{v} \cup_{A^{\prime}} V_{y}$ are Seifert fibered spaces over a disk with two singular fibers of indices $a, \alpha$ and $b, \beta$, respectively.

To determine how the fibers of $M_{1}$ and $M_{2}$ intersect at the boundary, we observe that:

- $V_{x}$ and $V_{y}$ lie on opposite sides of $T$ and intersect at $D_{w^{\prime}}$, and

- the annulus complementary to $A$ in $\partial V_{x}$ is given by $B \cup D_{w^{\prime}}$, while the annulus complementary to $A^{\prime}$ in $\partial V_{y}$ is given by $B^{\prime} \cup D_{w^{\prime}}$.

Now, it is clear that the cores of the annuli $B \cup D_{w^{\prime}} \subset \partial M_{1}$ and $B^{\prime} \cup D_{w^{\prime}} \subset$ $\partial M_{2}$ intersect in one point; in fact, the disk $D_{w^{\prime}}$ can be thought of as being a fat regular neighborhood of such a point of intersection; it follows that the fibers of $M_{1}$ and $M_{2}$ intersect in one point. The proof of Theorem 1.1 is now complete.

\section{Coherency invariants.}

In this section we prove Part (a) of Theorem 1.2; Part (b) is somewhat similar, and we refer the reader to $[\mathbf{1 4}]$ for a detailed proof. The determination of when a decomposition of a manifold $\mathcal{M}$ as in Theorem 1.1 gives rise to a t-manifold structure will depend on invariants of the Seifert fibered structures that detect the necessary coherent intersections. We begin by describing how these invariants are constructed via Seifert invariants. The set of notes by Jankins and Neumann [12] contain a nice exposition of basic facts about Seifert fibered spaces, some of which we present here to set the notation we will be using in the sequel.

Let $p: M \rightarrow D$ be an oriented Seifert fibered space over a disk with two singular fibers of indices $a_{1}, a_{2}$ and fixed orientation on all fibers. Denote by $x_{i}$ the projection point in $D$ of the singular fiber of index $a_{i}$.

Let $\alpha \subset D$ be a properly embedded arc separating $D$ into two disks $D_{1}, D_{2}$ with $x_{i} \in$ int $D_{i}$. The fibered annulus $A=p^{-1}(\alpha)$ then separates $M$ into two solid tori $V_{1}$ and $V_{2}$, with $p^{-1}\left(x_{i}\right) \subset V_{i}$. We take one of the components of $\partial A$ as representative of the regular fibers of $M$, and denote it by $h$. Let $D^{*}=D \backslash \operatorname{int}\left(D_{1}^{\prime} \cup D_{2}^{\prime}\right)$, where $D_{i}^{\prime} \subset D_{i}$ is a small disk containing $x_{i}$ in its interior, and let $M^{*}=p^{-1}\left(D^{*}\right)$ be the associated trivial $S^{1}$-bundle over $D^{*}$.

Assume now that an oriented simple closed curve $\mu \subset \partial M$ intersecting the fibers of $\partial M$ transversely in one point is given. Any section $s: D^{*} \rightarrow M^{*}$ of $p \mid M^{*}$ such that $s(\partial D)=\mu$ can be used to frame the torus $\partial V_{i}$ via the curves $\mu_{i}=s\left(\partial D_{i}\right)$ (framing meridians) and $h$ (longitude), where each $\mu_{i}$ inherits its orientation from $\mu$; we call $\mu_{i}$ a $\mu$-meridian of $V_{i}$. Observe that $\mu_{1} \cap \mu_{2}$ is an arc in $A$ receiving opposite orientations from the framing meridians $\mu_{1}, \mu_{2}$. To stress the dependency between the circle $\mu$ and the section $s$ used to frame $M$, we refer to such a framing as a $\mu$-framing of $M$. 
With respect to a particular $\mu$-framing, the meridian circle $m_{i} \subset \partial V_{i}$ of $V_{i}$ can be oriented so that, in $H_{1}\left(\partial V_{i}\right)$, it is of the form

$$
m_{i}=a_{i} \mu_{i}+p_{i} h, \quad a_{i}>0 .
$$

The ordered 4 -tuple $\left(a_{1}, p_{1} ; a_{2}, p_{2}\right)$ is called the Seifert invariant of $M$. The Euler number of the Seifert fibration on $M$ is defined as $e(M)=a_{1} / p_{1}+$ $a_{2} / p_{2}$. These invariants are sensitive to changes in the orientation of the manifold and/or the fibers, e.g., $e(-M)=-e(M)$, where $-M$ denotes the manifold $M$ with the opposite orientation.

Remark 4.1. The Seifert invariant also depends on the particular section used to construct it. In fact, if a different section is used, then the new Seifert invariant obtained will be of the form $\left(a_{1}, p_{1}+k_{1} a_{1} ; a_{2}, p_{2}+k_{2} a_{2}\right)$, where $k_{1}$ and $k_{2}$ are integers such that $k_{1}+k_{2}=0$ (see [12]). The Euler number of $M$, however, is independent of the section used to get the Seifert invariant.

The function $\llbracket . . . \rrbracket$ defined in the Introduction can now be used to construct an invariant of $M$ similar to its Euler number. If the Seifert invariant of $M$ with respect to some $\mu$-framing is $\left(a_{1}, p_{1} ; a_{2}, p_{2}\right)$, define

$$
c(M)=\llbracket p_{1} / a_{1} \rrbracket+\llbracket p_{2} / a_{2} \rrbracket .
$$

The periodicity of the function $\llbracket . . \rrbracket$ and Remark 4.1 immediately imply that $c(M)$ is an invariant of $M$; that is, like $e(M), c(M)$ depends only on the oriented Seifert fiber structure of $M$ and not on the particular Seifert invariant used in its construction.

Let $M, M^{\prime}$ be oriented Seifert fibered spaces over a disk with two singular fibers, and suppose that $\mathcal{M}=M \cup_{\partial} M^{\prime}$ in such a way that the fibers of $M, M^{\prime}$ at the boundary intersect in one point after an orientation reversing gluing. Under these conditions, the manifold $\mathcal{M}$ admits certain Heegaard splittings similar to the ones considered in the proof of Subcase 2(b) of Theorem 1.1 (Section 3).

Let $h \subset \partial M, h^{\prime} \subset \partial M^{\prime}$ be fibers intersecting in one point; we use these (oriented) fibers to induce framings on $M, M^{\prime}$ in the sense of the previous section. That is, $M$ is given an $h^{\prime}$-framing and $M^{\prime}$ and $h$-framing, and we choose our notation so that:

$$
\begin{gathered}
M=V_{1} \cup_{A} V_{2}, \\
\text { fiber }=h \subset \partial A, \\
\mu_{i}=h^{\prime} \text {-meridian of } \partial V_{i}, \\
m_{i}=a_{i} \mu_{i}+p_{i} h, a_{i}>1, \\
\mu_{i} \cdot h=1 \text { in } \partial V_{i},
\end{gathered}
$$

Seifert invariant $=\left(a_{1}, p_{1} ; a_{2}, p_{2}\right)$

$$
M^{\prime}=V_{1}^{\prime} \cup A^{\prime} V_{2}^{\prime},
$$$$
\text { fiber }=h^{\prime} \subset \partial A^{\prime} \text {, }
$$

$$
\text { and } \begin{gathered}
\mu_{i}^{\prime}=h \text {-meridian of } \partial V_{i}^{\prime}, \\
m_{i}^{\prime}=a_{i}^{\prime} \mu_{i}^{\prime}+p_{i}^{\prime} h^{\prime}, a_{i}^{\prime}>1, \\
\mu_{i}^{\prime} \cdot h^{\prime}=1 \text { in } \partial V_{i}^{\prime},
\end{gathered}
$$
Seifert invariant $=\left(a_{1}^{\prime}, p_{1}^{\prime} ; a_{2}^{\prime}, p_{2}^{\prime}\right)$.

Let $T$ be the embedded separating torus $\partial M=\partial M^{\prime}$ in $\mathcal{M}$. The four circles $\partial A \cup \partial A^{\prime}$ split $T$ into four squares, each of which represents one of 


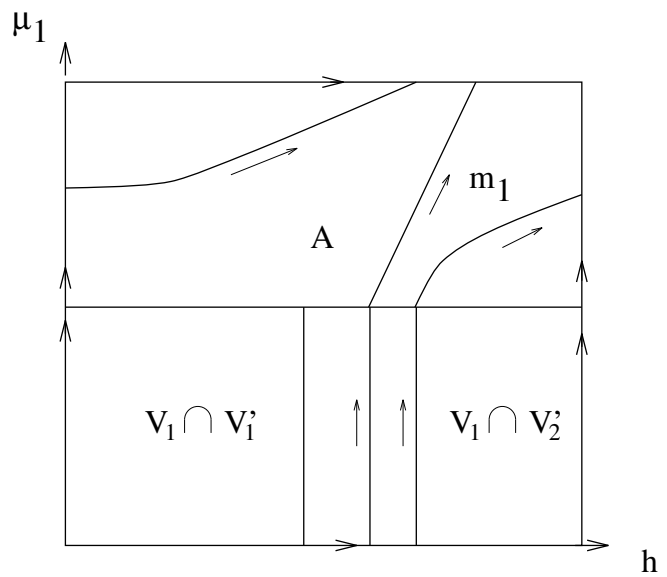

Figure 7. $m_{1} \subset \partial V_{1}$.

the intersections $V_{i} \cap V_{j}^{\prime}$ for $\{i, j\} \subset\{1,2\}$, as in Figure 8. Clearly, $\mathcal{M}$ admits the following genus two Heegaard splittings:

$$
\begin{aligned}
& \text { (a) } \mathcal{M}=\left(V_{1} \cup_{V_{1} \cap V_{1}^{\prime}} V_{1}^{\prime}\right) \cup_{\partial}\left(V_{2} \cup_{V_{2} \cap V_{2}^{\prime}} V_{2}^{\prime}\right) \text {, } \\
& \text { (b) } \mathcal{M}=\left(V_{1} \cup_{V_{1} \cap V_{2}^{\prime}} V_{2}^{\prime}\right) \cup_{\partial}\left(V_{2} \cup_{V_{2} \cap V_{1}^{\prime}} V_{1}^{\prime}\right) \text {. }
\end{aligned}
$$

We will call any genus two Heegaard splitting of $\mathcal{M}$ obtained in this way standard. Define the coherency invariant $\mathcal{C}(\mathcal{M})$ of $\mathcal{M}$ as

$$
\mathcal{C}(\mathcal{M})=c(M) c\left(M^{\prime}\right)=\left(\llbracket p_{1} / a_{1} \rrbracket+\llbracket p_{2} / a_{2} \rrbracket\right) \cdot\left(\llbracket p_{1}^{\prime} / a_{1}^{\prime} \rrbracket+\llbracket p_{2}^{\prime} / a_{2}^{\prime} \rrbracket\right) .
$$

Note that this invariant is independent of the orientations of $M, M^{\prime}$.

Proof of Theorem 1.2. We will show that any standard Heegaard splitting of the manifold $\mathcal{M}$ gives rise to a t-manifold structure on $\mathcal{M}$ iff $\mathcal{C}(\mathcal{M})<0$. To fix notation, we assume that the Heegaard splitting under consideration is of Type (a).

Let $H_{1}=V_{1} \cup V_{1}^{\prime}$ and $H_{2}=V_{2} \cup V_{2}^{\prime}$. We turn the meridian circles $m_{1}, m_{1}^{\prime}$ of $V_{1}, V_{1}^{\prime}$ into meridian circles of $H_{1}$ by isotoping them away from the square $V_{1} \cap V_{1}^{\prime}$; similarly, we isotope $m_{2}, m_{2}^{\prime}$ in $H_{2}$ away from the square $V_{2} \cap V_{2}^{\prime}$. Figure 7 shows $m_{1} \subset \partial V_{1}$ with all regions involved.

Remark 4.2. Observe that, as $m_{1}=a_{1} \mu_{1}+p_{1} h^{\prime}$ and $a_{1}>0$, the meridian circle $m_{1}$ and $\mu_{1}$ (which is essentially $h^{\prime}$ ) both intersect $h$ in the same direction. A similar remark applies to the other meridians.

Consider now the genus two handlebody $H_{1}$ with meridian circles $m_{1}, m_{1}^{\prime}$ and attaching curves $m_{2}, m_{2}^{\prime} \subset \partial H_{1}$. In order for $\mathcal{M}$ to get a t-manifold structure from this Heegaard diagram, the intersections between the attaching curves and the meridian circles of $H_{1}$ must be coherent and represent all types. We first observe that: 


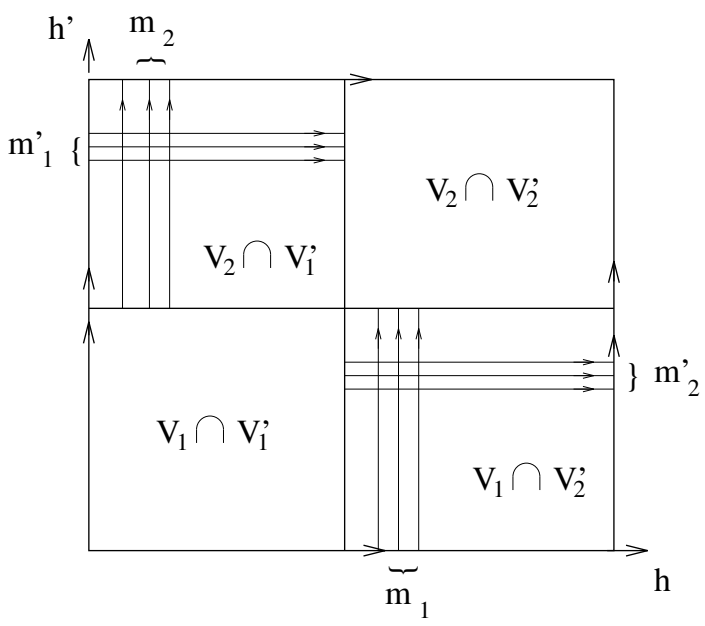

Figure 8. $m_{1}^{\prime}, m_{2}$ and $m_{1}, m_{2}^{\prime}$ always intersect coherently.

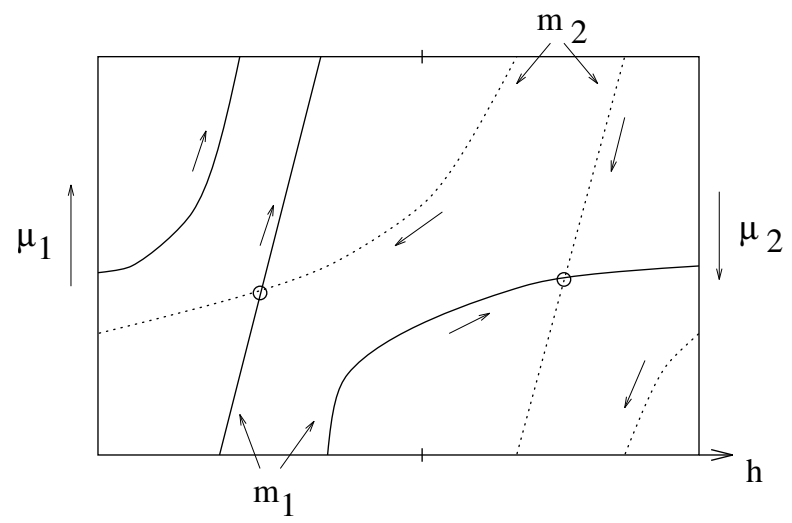

Figure 9. The arcs of $m_{1}, m_{2}$ in the annulus $A$.

- All the intersections of $m_{1}$ with $m_{2}$ occur in the annulus $A$, while all the intersections of $m_{1}$ with $m_{2}^{\prime}$ occur in the square $V_{1} \cap V_{2}^{\prime}$, and

- all the intersections of $m_{1}^{\prime}$ with $m_{2}^{\prime}$ occur in the annulus $A^{\prime}$, while all the intersections of $m_{1}^{\prime}$ with $m_{2}$ occur in the square $V_{1}^{\prime} \cap V_{2}$.

From the way the meridians intersect the square regions, it follows that such intersections must necessarily be coherent, as in Figure 8. The orientations of the arcs shown in Figure 8 were obtained from our observations in Remark 4.2. Hence, the signs of the intersections within the squares depend only on the framings induced by $h \cup h^{\prime}$ on $M, M^{\prime}$.

The intersections occurring in the annuli $A$ and $A^{\prime}$ are a bit more involved. Figure 9 shows how $m_{1}$ and $m_{2}$ may intersect in $A \subset V_{1}$. Observe that, 
since $m_{1}$ and $m_{2}$ do not intersect the squares $V_{1} \cap V_{1}^{\prime}$ and $V_{2} \cap V_{2}^{\prime}$, they can only intersect $A$ in a restricted way which may give rise to intersections of opposite signs (i.e., incoherent intersections).

To study this coherency problem, we first turn $A$ into a torus $T_{A}$ by gluing the boundary circles of $A$ in such a way that the arcs $\mu_{1} \cap A, m_{1} \cap A$, and $m_{2} \cap A$ each give rise to a closed curve in $T_{A}$. (Equivalently, we collapse the annulus in $\partial V_{1}$ complementary to $A$ onto $h$ to get $T_{A}$.) We call the resulting curves $\mu_{1}\left|A, m_{1}\right| A, m_{2} \mid A$, respectively, and orient them following the orientation of the arcs used in their construction. As framing for $T_{A}$, we take the circles $\mu_{1} \mid A \subset T_{A}$ and $h \subset T_{A}$ with $\mu_{1} \mid A \cdot h=1$. In this way, since the framed tori $T_{A}$ and $\partial V_{1}$ are essentially 'the same', we have that

$$
m_{1}\left|A=a_{1} \mu_{1}\right| A+p_{1} h,
$$

and since the two $\operatorname{arcs} \mu_{1} \cap A$ and $\mu_{2} \cap A$ agree but have opposite orientations in $A$ (see Figure 9 ), then

$$
m_{2}\left|A=-a_{2} \mu_{1}\right| A+p_{2} h .
$$

We further observe that $h \subset T_{A}$ naturally splits into two $\operatorname{arcs} h_{1}, h_{2}$, such that $m_{1} \mid A$ intersects $h$ only in $h_{1}$ and $m_{2} \mid A$ intersects $h$ only in $h_{2}$. Now, it is clear that

$m_{1}, m_{2}$ intersect coherently in $A$ iff $m_{1}\left|A, m_{2}\right| A$ intersect coherently in $T_{A}$.

We abstract the present situation in the form of the following lemma characterizing coherent intersections of embedded curves in a torus relative to a fixed curve:

Lemma 4.3. Let $T$ be a torus with oriented meridian-longitude curves $\mu$ and $\lambda$. Suppose that $\lambda$ is the union of two arcs $\lambda_{1}, \lambda_{2}$, and that two embedded circles $\gamma_{1}, \gamma_{2}$ in $T$ are given such that $\gamma_{i}$ intersects $\lambda$ in int $\left(\lambda_{i}\right)$ only, for $i=1,2$. Subject only to this constraint, we further assume that $\gamma_{1}$ and $\gamma_{2}$ intersect minimally.

If $\gamma_{i}=a_{i} \mu+p_{i} \lambda$ and $a_{i} \geq 2$, for $i=1,2$, then $\gamma_{1}$ and $\gamma_{2}$ intersect coherently iff $\llbracket p_{1} / a_{1} \rrbracket \neq \llbracket p_{2} / a_{2} \rrbracket$. More precisely, we have that $\gamma_{1}$ and $\gamma_{2}$ intersect coherently with $\gamma_{1} \cdot \gamma_{2}>0$ iff $\llbracket p_{2} / a_{2} \rrbracket>\llbracket p_{1} / a_{1} \rrbracket$.

We remark that the conditions given in the lemma are independent of the meridian circle $\mu$ used to specify coordinates in $T$. The proof of the lemma is given at the end of this section, and we use it now to study the coherent intersection of the circles $m_{1}, m_{2}$ and $m_{1}^{\prime}, m_{2}^{\prime}$ in the annuli $A$ and $A^{\prime}$, respectively. For such coherent intersections to represent all types, we must have that either:

(i) $m_{2}$ and $m_{2}^{\prime}$ intersect $m_{1}$ in the same direction and $m_{1}^{\prime}$ in opposite directions, or 
(ii) $m_{2}$ and $m_{2}^{\prime}$ intersect $m_{1}^{\prime}$ in the same direction and $m_{1}$ in opposite directions.

We deal with (i) first, and refer to the frames of $\partial V_{1}$ and $\partial V_{1}^{\prime}$ to compute all intersection numbers. From Figure 8 above, we can see that

$$
m_{1} \cdot m_{2}^{\prime}>0 \text { and } m_{1}^{\prime} \cdot m_{2}>0
$$

therefore, (i) is equivalent to:

(i') $m_{1} \cdot m_{2}>0$ and $m_{1}^{\prime} \cdot m_{2}^{\prime}<0$.

The coherent intersections of $m_{1}, m_{2}$ that satisfy (i') can be handled from the point of view of the framed torus $T_{A}$ and the curves $m_{1}\left|A, m_{2}\right|_{A}$ via Lemma 4.3. To apply the lemma, though, the $\mu_{1}$-coefficients of both curves must be positive to begin with. This is only a problem for $m_{2} \mid A$, easily corrected by rephrasing ( $\left.\mathrm{i}^{\prime}\right)$ in terms of $-m_{2} \mid A$; that is,

$$
\begin{aligned}
& \begin{array}{c}
m_{1}, m_{2} \text { intersect coherently } \\
\text { and } m_{1} \cdot m_{2}>0
\end{array} \text { iff } \begin{array}{c}
m_{1}\left|A, m_{2}\right| A \text { intersect coherently } \\
\text { and } m_{1} \mid A \cdot\left(-m_{2} \mid A\right)<0
\end{array} \\
& \text { iff } \llbracket-p_{2} / a_{2} \rrbracket<\llbracket p_{1} / a_{1} \rrbracket \\
& \text { iff } \quad c(M)=\llbracket p_{1} / a_{1} \rrbracket+\llbracket p_{2} / a_{2} \rrbracket>0 .
\end{aligned}
$$

Similarly, $m_{1}^{\prime}, m_{2}^{\prime}$ intersect coherently and $m_{1}^{\prime} \cdot m_{2}^{\prime}<0$ iff $c\left(M^{\prime}\right)<0$. Since it is now apparent that (ii) is equivalent to $c(M)<0$ and $c\left(M^{\prime}\right)>0$, we conclude that the manifold $\mathcal{M}$ gets a t-manifold structure from the given Heegaard splitting iff $\mathcal{C}(\mathcal{M})=c(M) c\left(M^{\prime}\right)<0$. The Heegaard splitting (b) can be handled in a similar way and yields the same conclusion. The theorem follows.

Proof of Lemma 4.3. Let $A$ be the annulus obtained by cutting $T$ along $\lambda$, and consider the arcs of $\gamma_{i}$ in $A$. Call any two such arcs $\lambda_{i}$-parallel if they cobound a band in $A$ that is disjoint from the two copies of $\lambda_{j}(j \neq i)$ in $\partial A$. There can be simultaneously at most two disjoint arcs that are not $\lambda_{i}$-parallel. Since $a_{i} \geq 2$, it follows that the arcs of $\gamma_{i}$ in $A$ must split into two non $\lambda_{i}$-parallel families of $\lambda_{i}$-parallel arcs; amalgamate each of these families into one arc, and call the resulting two $\operatorname{arcs} x_{i}$ and $y_{i}$. These two arcs give rise to a closed curve in $T$ in the obvious way, which we denote by $\bar{\gamma}_{i}$ and orient so as to follow the orientation of the $\operatorname{arcs} x_{i}, y_{i}$. The new curves $\bar{\gamma}_{i}$ 's share the following properties in common with the $\gamma_{i}$ 's:

- $\gamma_{1}, \gamma_{2}$ intersect coherently iff $\bar{\gamma}_{1}, \bar{\gamma}_{2}$ intersect coherently;

- in the case of coherent intersections, $\gamma_{1} \cdot \gamma_{2}$ and $\bar{\gamma}_{1} \cdot \bar{\gamma}_{2}$ have the same sign.

Both properties follow easily from the way the $\bar{\gamma}_{i}$ 's were constructed. We now claim that: 


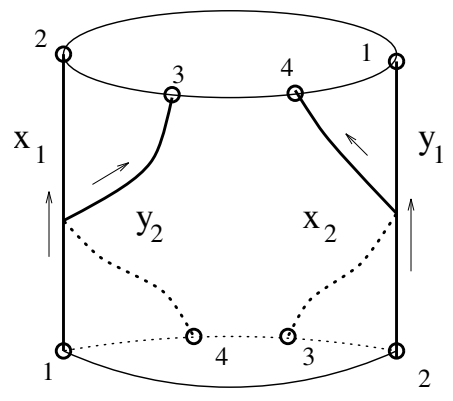

A

(a)

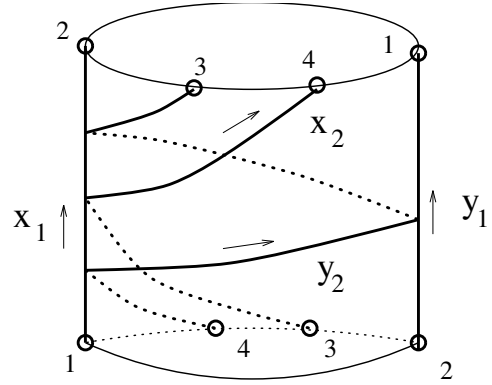

A

(b)

Figure 10. The $\operatorname{arcs} x_{i}$ and $y_{i}$ in the annulus $A$.

Claim. $\bar{\gamma}_{1}$ and $\bar{\gamma}_{2}$ intersect coherently iff their intersection number in $T$ is nonzero.

Proof. To see this, let $x_{i}, y_{i}$ be the two $\operatorname{arcs}$ of $\bar{\gamma}_{i}$ in $A$. We proceed according to the following two cases:

Case 1. No two arcs of $\bar{\gamma}_{1}, \bar{\gamma}_{2}$ in $A$ intersect in more than one point.

In this case, the situation must be as in Figure 10(a), where clearly the intersection number of the curves is zero and the intersection is not coherent.

Case 2. One arc of $\bar{\gamma}_{1}$ intersects an arc of $\bar{\gamma}_{2}$ in at least two points.

Suppose $\left|x_{1} \cap x_{2}\right| \geq 2$; Figure 10(b) shows the annulus $A$ and all the arcs involved. Observe that the arc $x_{2}$ must intersect $x_{1}$ and $y_{1}$ in the same direction as $y_{2}$, inducing coherent intersections (and hence nontrivial intersection number). This proves our claim.

To translate the information obtained about $\bar{\gamma}_{1}$ and $\bar{\gamma}_{2}$ in terms of $\gamma_{1}$ and $\gamma_{2}$, we proceed as follows. Choose the notation so that $\min \left\{\left|x_{i} \cap \mu\right|,\left|y_{i} \cap \mu\right|\right\}=$ $\left|x_{i} \cap \mu\right|=n_{i}$. In order to construct the closed curve $\bar{\gamma}_{i}$, the arc $y_{i}$ must be of the form $x_{i} \pm \lambda$, and so $\left|y_{i} \cap \mu\right|=\left|x_{i} \cap \mu\right|+1=n_{i}+1$. Therefore,

$$
\bar{\gamma}_{i}=2 \mu+\operatorname{sgn}\left(p_{i}\right)\left(2 n_{i}+1\right) \lambda .
$$

Now suppose that $a_{i}^{\prime}$ copies of $x_{i}$ and $a_{i}^{\prime \prime}$ copies of $y_{i}$ are needed to construct $\gamma_{i}$, where $a_{i}^{\prime}+a_{i}^{\prime \prime}=a_{i}$. Then,

$$
\begin{aligned}
\gamma_{i} & =a_{i} \mu+p_{i} \lambda \\
& =\left(a_{i}^{\prime}+a_{i}^{\prime \prime}\right) \mu_{i}+\operatorname{sgn}\left(p_{i}\right)\left(n_{i} a_{i}^{\prime}+\left(n_{i}+1\right) a_{i}^{\prime \prime}\right) \lambda \\
& =a_{i} \mu+\operatorname{sgn}\left(p_{i}\right)\left(n_{i} a_{i}+a_{i}^{\prime \prime}\right) \lambda
\end{aligned}
$$


whence $p_{i}=\operatorname{sgn}\left(p_{i}\right)\left(n_{i} a_{i}+a_{i}^{\prime \prime}\right)$, and so $n_{i}=\left[\left|p_{i} / a_{i}\right|\right]$, where the brackets denote the greatest integer function. Observing that $2 \llbracket x \rrbracket=\operatorname{sgn}(x)(2[|x|]+1)$ for $x$ not an integer, we can write

$$
\begin{aligned}
\bar{\gamma}_{1} \cdot \bar{\gamma}_{2} \neq 0 \text { iff } & \operatorname{sgn}\left(p_{1}\right)\left(2 n_{1}+1\right) \neq \operatorname{sgn}\left(p_{2}\right)\left(2 n_{2}+1\right) \\
\text { iff } & \llbracket p_{1} / a_{1} \rrbracket \neq \llbracket p_{2} / a_{2} \rrbracket
\end{aligned}
$$

and

$$
\begin{aligned}
\bar{\gamma}_{1} \cdot \bar{\gamma}_{2}>0 & \text { iff } \operatorname{sgn}\left(p_{2}\right)\left(2 n_{2}+1\right)-\operatorname{sgn}\left(p_{1}\right)\left(2 n_{1}+1\right)>0 \\
& \text { iff } \llbracket p_{2} / a_{2} \rrbracket>\llbracket p_{1} / a_{1} \rrbracket .
\end{aligned}
$$

The lemma follows.

\section{Proper matrices.}

In this section, we introduce some basic properties of proper matrices and give a proof of Theorem 1.3.

Two $k \times n$ matrices are said to be t-equivalent if one can be obtained from the other by performing a finite sequence of operations of the following types:

(a) Permutating any row (column, resp.) with any other row (column, resp.);

(b) multiplying any row or column by \pm 1 ;

(c) substituting any nonzero entry by any nonzero real number of the same sign.

These operations will be referred to as t-equivalences of Types (a), (b), and (c). Observe that if $A$ and $A^{\prime}$ are t-equivalent, then $A$ is proper iff $A^{\prime}$ is proper; this fact will be used frequently. Given vectors $v^{\prime} \in \mathbb{R}^{s}$ and $v^{\prime \prime} \in \mathbb{R}^{n-s}$, we use the notation $\left(v^{\prime} \mid v^{\prime \prime}\right)$ to represent the vector in $\mathbb{R}^{n}$ obtained by taking the entries of $v^{\prime}$ followed by those of $v^{\prime \prime}$.

Proof of Theorem 1.3 (cf. [9, Lemma 4.4]). Assume (c) does not hold, and let $v$ be a nonzero vector in $\mathbb{R}^{n}$ which is orthogonal to all the rows of $A$. Let $a$ be a row of $A$ that represents $v$. If all the entries of $v$ are nonzero then $a$ must be the zero vector, since $\langle v, a\rangle=0$. As the zero vector represents all types, either $A$ consists of a row of zeroes and satisfies (a), or else it must satisfy (b). Hence, passing to a t-equivalent matrix if necessary, we may assume that $v$ is of the form $\left(O \mid v^{\prime \prime}\right)$, where, for some $0<s<n$, all the entries of $v^{\prime \prime} \in \mathbb{R}^{n-s}$ are nonzero, and $O \in \mathbb{R}^{s}$ is the zero vector.

Let $u^{\prime}$ be any vector in $\mathbb{R}^{s}$, and consider the vector $u=\left(u^{\prime} \mid v^{\prime \prime}\right)$. If $a=\left(a^{\prime} \mid a^{\prime \prime}\right)$ is any row of $A$ that represents the vector $u$, then $a^{\prime \prime}=O$ since $\langle v, a\rangle=0$. Hence, the matrix $B$ whose rows are the rows of $A$ of the form $\left(a^{\prime} \mid O\right), O \in \mathbb{R}^{n-s}$, represents all $n$-types. If $A=B$ then (a) holds, otherwise (b) holds. 
In the context of proper matrices, Parry's Theorem [13] can be restated as follows:

Lemma 5.1 (Parry's Theorem Restated). Let $A$ be an integral $k \times n$ proper matrix. Then there is an $n \times n$ submatrix $A_{0}$ of $A$ such that $\left|\operatorname{det}\left(A_{0}\right)\right| \geq 2$.

Proof. By Parry's Theorem, for some subset $S$ of the rows of $A$, the group $G_{S}=\mathbb{Z}^{n} /\langle S\rangle$ has nontrivial torsion. Let $S^{\prime}$ be a maximal linearly independent subset of $S$; then $G_{S}^{\prime}=\mathbb{Z}^{n} /\left\langle S^{\prime}\right\rangle$ also has nontrivial torsion. Since $A$ is proper, $\operatorname{rank}(A)=n$ and so there is a set $T$ which consists of $n$ linearly independent rows of $A$ and contains $S^{\prime}$. Since the group $G_{T}=\mathbb{Z}^{n} /\langle T\rangle$ necessarily has nontrivial torsion (and is finite), the matrix $A_{0}$ whose rows are the vectors in $T$ satisfies the conclusion of the lemma.

In the next lemma, we give a sufficient condition for a matrix to represent all types in terms of t-equivalence.

Lemma 5.2. Let $A$ be a $k \times n$ matrix. If any matrix that is t-equivalent to $A$ has rank $n$, then $A$ represents all types.

Proof. Suppose $A$ fails to represent some vector $v \in \mathbb{R}^{n}$; without loss of generality, and after a t-equivalence if necessary, we may assume that $v=$ $(1,1, \ldots, 1)$. Then every row of $A$ must have two entries with opposite signs. If $a$ is any row of $A$, let $a^{*}$ be a row vector obtained by substituting a pair of oppositely signed nonzero entries of $a$ with a pair of nonzero real numbers of the same signs, respectively, such that the sum of the entries of $a^{*}$ is zero, and let $A^{*}$ be the matrix whose rows are the $a^{*}$ 's. Then clearly $A^{*}$ is t-equivalent to $A$, but $\operatorname{rank}\left(A^{*}\right)<n$ since the column vectors of $A^{*}$ add up to zero. The lemma follows.

For any nonsingular $n \times n$ matrix $A=\left(a_{i j}\right)$, we say that $\operatorname{det}(A)$ is coherent if all the nonzero terms in the usual expansion $\sum_{\sigma} \operatorname{sgn}(\sigma) a_{1 \sigma(1)} \cdots a_{n \sigma(n)}$ of $\operatorname{det}(A)$ have the same sign.

Lemma 5.3. Let $A$ be an $n \times n$ nonsingular matrix. Then every matrix that is t-equivalent to $A$ is nonsingular iff $\operatorname{det}(A)$ is coherent.

Proof. Suppose $\operatorname{det}(A)$ is not coherent, say two terms $\operatorname{sgn}(\sigma) a_{1 \sigma(1)} \ldots a_{n \sigma(n)}$ and $\operatorname{sgn}\left(\sigma^{\prime}\right) a_{1 \sigma^{\prime}(1)} \ldots a_{n \sigma^{\prime}(n)}$ in the expansion of $\operatorname{det}(A)$ are nonzero and have opposite signs. Fix the numbers $a_{1 \sigma(1)}, \ldots, a_{n \sigma(n)}$, and replace each of the other nonzero entries of $A$ by a sufficiently small number of the same sign. Obtain in this way a matrix $B$ that is t-equivalent to $A$, and such that $\operatorname{det}(B)$ and $\operatorname{sgn}(\sigma) a_{1 \sigma(1)} \ldots a_{n \sigma(n)}$ have the same sign. In a similar way, we can find a matrix $B^{\prime}$ which is t-equivalent to $A$ and such that $\operatorname{det}\left(B^{\prime}\right)$ and $\operatorname{sgn}\left(\sigma^{\prime}\right) a_{1 \sigma^{\prime}(1)} \ldots a_{n \sigma^{\prime}(n)}$ have the same sign. Since the set $\mathcal{A}$ of all matrices which are t-equivalent to $A$ is connected (homeomorphic to $\mathbb{R}^{k}$, where $k$ 
is the number of nonzero entries of $A$ ) and the determinant function is continuous on $\mathcal{A}$, it follows that $\operatorname{det}\left(A_{0}\right)=0$ for some $A_{0} \in \mathcal{A}$.

Conversely, suppose that $A$ is nonsingular and $\operatorname{det}(A)$ is coherent, and let $A^{\prime}$ be t-equivalent to $A$. Then there is a matrix $A^{\prime \prime}$ such that:

(i) $A^{\prime \prime}$ can be obtained via Type (c) t-equivalences on $A$, and

(ii) $A^{\prime \prime}$ can be obtained via Type (a) or (b) t-equivalences on $A^{\prime}$.

It follows from (i) that $\operatorname{det}\left(A^{\prime \prime}\right)$ is coherent and hence that $A^{\prime \prime}$ is nonsingular. Since $\left|\operatorname{det}\left(A^{\prime}\right)\right|=\left|\operatorname{det}\left(A^{\prime \prime}\right)\right|$ by (ii), $A^{\prime}$ is nonsingular as claimed.

Remark 5.4. Implicit in Lemma 5.3 is the fact that, for nonsingular matrices, the property of having coherent determinant is invariant under tequivalence.

Recall that a matrix is proper if it represents all types and does not satisfy (a) or (b) of Theorem 1.3. More generally, we say that a matrix $A$ represents types properly, and that it is t-proper for short, if each row of $A$ represents some type which is not represented by any other row of $A$. Clearly, proper matrices are t-proper, but the converse, in general, does not hold; observe however that a matrix is proper iff it is t-proper and represents all types. In this context, the previous two lemmas combine to prove the following result:

Corollary 5.5. Let $A$ be a nonsingular t-proper square matrix. Then $A$ is proper iff $\operatorname{det}(A)$ is coherent.

The next result is a mild generalization of Parry's Theorem for square matrices.

Lemma 5.6. Let $A$ be a nonsingular $n \times n$ matrix $(n \geq 2)$ with integral entries and coherent determinant. Then $|\operatorname{det}(A)| \geq 2$.

Proof. If $n=2$ then $A$ is t-equivalent to a matrix of the form $\left(\begin{array}{c}++ \\ +\end{array}\right)$ by performing only Type (a) or (b) t-equivalences; hence $|\operatorname{det}(A)| \geq 2$. Proceeding by induction, suppose that $A=\left(a_{i j}\right)$ is a nonsingular $n \times n$ matrix with $n>2$ and coherent determinant. After performing Type (a) or (b) t-equivalences on $A$ if necessary, we may assume that both $a_{11}$ and its cofactor $C_{11}$ are nonzero. Let $M_{11}$ be the $(n-1) \times(n-1)$ minor corresponding to $a_{11}$, so that $\operatorname{det}\left(M_{11}\right)=C_{11}$. Since $\operatorname{det}(A)=a_{11} C_{11}+\ldots$ is coherent (see Remark 5.4), it follows that $\operatorname{det}\left(M_{11}\right)$ is also coherent; as $M_{11}$ is nonsingular we get that $\left|\operatorname{det}\left(M_{11}\right)\right| \geq 2$ by the induction hypothesis, and hence that $|\operatorname{det}(A)| \geq 2$ by coherency of $\operatorname{det}(A)$.

A matrix that represent all types is proper iff it is t-proper. In the case of square matrices, it is possible to replace the later condition by one that involves only some knowledge about the 0-girth of the matrix. This is the content of Theorem 1.5, whose proof is given below. We point out that the 0 -girth of a square matrix is invariant under t-equivalence, a fact which is used implicitly in the proof. 
Proof of Theorem 1.5. Suppose $A$ represents all types but is not proper; then, by definition, $A$ must either have a column of zeroes or some proper subset of its rows must represent all types. In the first case the 0-girth of $A$ is at least $n+1$ (the girth of any zero-column); in the second case, let $A_{0}$ be a $k \times n$ submatrix of $A$ that represents all types, with $k<n$ smallest among all choices. After a t-equivalence, if necessary, we may assume that $A_{0}=(B \mid O)$, where $B$ is a $k \times l$ matrix which represents all types and has no zero columns. The minimality of $k$ then implies that $B$ is proper and hence that $l \leq k$ by Theorem 1.3. Since $A$ is t-equivalent to a matrix of the form

$$
\left(\begin{array}{cc}
B & O \\
C & D
\end{array}\right), O=\text { zero matrix }
$$

the 0 -girth of $A$ is at least $k+(n-l) \geq n$. Hence, in all cases, the 0-girth of $A$ is greater than $n-1$.

For the converse, assume that $A$ is proper. If the 0 -girth of $A$ is at least $n$ then, after performing a t-equivalence if necessary, $A$ may be assumed to be of the form given in (5.1), where $B$ is a $k \times l$ matrix with $l \leq k<n$; observe that $k=l>0$ since $A$ is nonsingular, so $B$ and $D$ are square matrices. Since $A$ is proper, each matrix t-equivalent to $A$ is proper and hence nonsingular. From the equation $\operatorname{det}(A)=\operatorname{det}(B) \operatorname{det}(D)$ we can then see that $B$ and every matrix t-equivalent to $B$ must also be nonsingular. Hence, by Lemma 5.2, $B$ represents all types, contradicting the properness of $A$.

The $n \times n$ matrix

$$
A_{n}=\left(\begin{array}{cccccc}
+ & + & \ldots & \ldots & + & + \\
+ & + & \ldots & \ldots & + & - \\
+ & + & \ldots & \ldots & - & 0 \\
\vdots & & & & & \vdots \\
+ & + & - & 0 & \ldots & 0 \\
+ & - & 0 & \ldots & \ldots & 0
\end{array}\right)
$$

where the signs of the nonzero entries are as shown, is proper and its 0-girth is $n-1$, which shows that the upper bound for the 0-girth of a proper matrix given in Theorem 1.5 is the best possible.

Combining Theorem 1.5 with Lemmas 5.2 and 5.3, we get the following characterization of proper square matrices:

Corollary 5.7. Let $A$ be an $n \times n$ nonsingular matrix, $n \geq 2$. Then $A$ is proper iff $\operatorname{det}(A)$ is coherent and the 0-girth of $A$ is at most $n-1$.

Corollary 5.8. Let $A$ be a square matrix. Then $A$ is proper iff $A^{T}$ is proper.

The family of proper square matrices $A_{n}$ given above is part of the bigger family of proper square matrices that have $2 \times 2$ submatrices not representing 
all types. We will see shortly that every member of this bigger family also has maximal 0-girth. The first step in this direction consists in showing that singular square matrices which remain singular under t-equivalences must have 'large' 0-girths.

Lemma 5.9. Let $A$ be an $n \times n$ matrix. Then the 0-girth of $A$ is at least $n+1$ iff every matrix that is t-equivalent to $A$ is singular.

This is a well-known result in combinatorics which goes back to P. Hall's Theorem [10] and the the marriage problem; a short proof of Hall's Theorem can be found in the paper of Halmos and Vaughn [11], and a proof of Lemma 5.9 can be found in the book of C. Berge [1, Theorem 9, p. 105].

We now restrict our attention to the family of proper square matrices that have $2 \times 2$ submatrices not representing all types.

Lemma 5.10. Let $A$ be an $n \times n$ proper matrix with $n>2$. If some $2 \times 2$ submatrix of $A$ does not represent all types, then the 0-girth of $A$ is $n-1$.

Proof. After a t-equivalence, if necessary, $A$ may be assumed to be of the form

$$
\left(\begin{array}{ccc}
a & b & \ldots \\
c & d & \ldots \\
\vdots & \vdots & B
\end{array}\right),
$$

where $a, b, c, d$ are all positive real numbers and $B$ is an $(n-2) \times(n-2)$ matrix. Then $\operatorname{det}(A)=(a d-b c) \operatorname{det}(B)+k_{1} a+k_{2} b+k_{3} c+k_{4} d+k$, where the numbers $\operatorname{det}(B), k_{1}, k_{2}, k_{3}, k_{4}$, and $k$ do not depend on $a, b, c$, or $d$.

After some some t-equivalence of $A$, if necessary, we may assume that $\operatorname{det}(B) \geq 0$. For any positive real number $x$, let $A_{x,+}$ and $A_{x,-}$ be the matrices t-equivalent to $A$ obtained by assigning the values $a=x, b=1, c=$ $1, d=x$ and $a=1, b=x, c=x, d=1$, respectively. If $\operatorname{det}(B)>0$ then

$$
\lim _{x \rightarrow \infty} \operatorname{det}\left(A_{x,+}\right)=\infty \text { and } \lim _{x \rightarrow \infty} \operatorname{det}\left(A_{x,-}\right)=-\infty,
$$

so that $\operatorname{det}(A)$ must be zero at some 'point' of the connected set $\mathbb{R}_{+}^{4}=$ $\{(a, b, c, d) \mid a, b, c, d>0\}$, which, in light of Theorem 1.3 and the invariance of properness under t-equivalences, contradicts the properness of $A$. Hence $\operatorname{det}(B)=0$, and similarly $\operatorname{det}\left(B^{\prime}\right)=0$ whenever $B^{\prime}$ is t-equivalent to $B$, which by Lemma 5.9 implies that the 0 -girth of the $(n-2) \times(n-2)$ matrix $B$, and hence of $A$, must be at least $n-1$. That $A$ has 0 -girth exactly equal to $n-1$ now follows from Theorem 1.5.

A word about standard notation in combinatorial matrix theory is appropriate now (cf. [2]). An $n \times n$ matrix $A$ is sign-nonsingular if $\operatorname{det}(A)$ is coherent (equivalently, if $|\operatorname{per}(A)|=|\operatorname{det}(A)|$, where $\operatorname{per}(A)$ denotes the permanent of $A$ ); if $A$ is a $(0,1)$-matrix, then $A$ is indecomposable if its 0girth is at most $n-1$. Thus, any nonsingular $(0,1)$-matrix is proper iff it is 


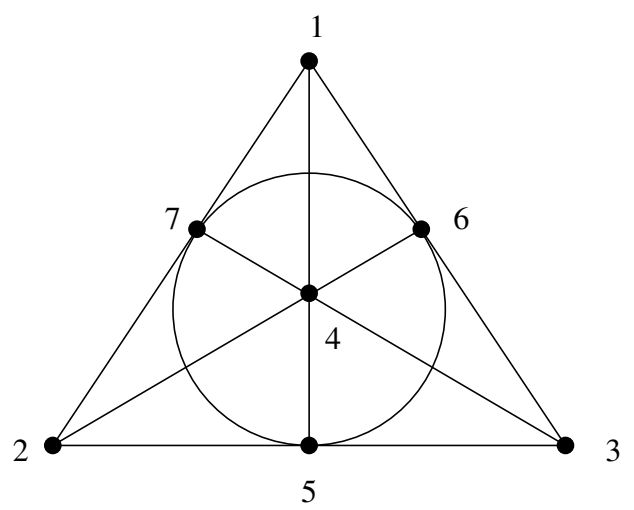

Figure 11. Lines in $X=\boldsymbol{P}^{2}\left(\mathbb{F}_{2}\right)$.

sign-nonsingular and indecomposable (Corollary 5.7), and any square matrix that represents all types is proper iff it is indecomposable (Theorem 1.5).

A finite projective plane of order $q \geq 1$ is an $n \times n(0,1)$-matrix $A$ that satisfies the equation $A A^{T}=q I+J$, where $n=q^{2}+q+1$ and $J$ is the matrix all of whose entries are 1's (cf. [2, §1.3]). The smallest projective plane corresponds to the value $q=1$ and is given by the proper matrix

$$
\left(\begin{array}{lll}
1 & 1 & 0 \\
1 & 0 & 1 \\
0 & 1 & 1
\end{array}\right)
$$

When $q$ is the power of a prime, an example of such a matrix can be constructed using the points and lines of the projective plane $\boldsymbol{P}^{2}\left(\mathbb{F}_{q}\right)$, where $\mathbb{F}_{q}$ is the field of $q$ elements. For instance, in the case $q=2$, there is only one finite projective plane (up to permutation of rows or columns), obtained as follows:

Let $X=\boldsymbol{P}^{2}\left(\mathbb{F}_{2}\right)$ denote the projective space of the 3 -dimensional vector space $\mathbb{F}_{2}^{3}$ over the field $\mathbb{F}_{2}$ of two elements. The space $X$ consists of 7 points which are organized into subsets that represent the lines of $X$ (the images of 2-dimensional subspaces of $\mathbb{F}_{2}^{3}$ ). The set $X$ is shown in Figure 11, where each point of $X$ has been identified with an integer from 1 to 7 ; any three points connected by a line or circle form a line in $X$. For each line $l=\{i, j, k\}$, $i<j<k$, of $X$, let [l] be the ordered 7-tuple consisting of 0's and +'s, whose +-entries occur at positions $i, j, k$, and let $A_{X}$ be the matrix whose rows are the vectors $[l]$ obtained in this way. The matrix $A_{X}$ is called the 
incidence matrix of $X$. Hence, up to t-equivalence,

$$
A_{X}=\left(\begin{array}{ccccccc}
0 & + & + & 0 & + & 0 & 0 \\
+ & 0 & + & 0 & 0 & + & 0 \\
+ & + & 0 & 0 & 0 & 0 & + \\
0 & 0 & 0 & 0 & + & + & + \\
+ & 0 & 0 & + & + & 0 & 0 \\
0 & + & 0 & + & 0 & + & 0 \\
0 & 0 & + & + & 0 & 0 & +
\end{array}\right)
$$

We can see that $A_{X}$ is proper as follows: Let $\varepsilon$ be any 7-type, and color red and green its positive and negative entries, respectively. Then $A_{X}$ represents $\varepsilon$ if a set of three like-colored entries of $\varepsilon$ correspond with the locations of + 's in some row of $A_{X}$. Suppose $\varepsilon$ has three red-colored entries which do not correspond to a the +'s in any row of $A_{X}$, say they correspond to the subset $1,2,3$ of $X$ (which is not a line). Then if any of the entries of $\varepsilon$ in positions 5,6 , or 7 is colored red we are done, for the sets $1,2,7,2,3,5$, and 1,3,6 are lines of $X$. Otherwise, all three positions 5,6, and 7 of $\varepsilon$ must be colored green and again we are done. By homogeneity of the space $X$, the same argument must work in all other cases, which proves that $A_{X}$ represents all types. That $A_{X}$ is proper now follows from Theorem 1.5 since the 0-girth of $A_{X}$ is 5 ; in particular, the results in this section imply that $A_{X}$ is sign-nonsingular, a property deemed 'unusual' in $[2, \S 1.3]$.

Higher order finite projective planes do not share the properness nor the sign-nonsingularity of the small cases $q \leq 2$; we leave the details of this fact to the interested reader, in the form of an exercise:

Exercise 5.11. Let $A$ be a finite projective plane of order $q \geq 1$. Then:

(a) $A$ is indecomposable; in fact, the 0 -girth of $A$ is $q^{2}+1$.

(b) $A$ represents all types (equivalently, $A$ is sign-nonsingular) iff $q \leq 2$.

\section{Topology of t-manifolds.}

We now apply the results on proper matrices and representation of types established in the previous section to the study of general t-manifolds. Let $M$ denote a t-manifold with a genus $n$ t-manifold structure $\boldsymbol{S}=\left(H_{n}, \boldsymbol{D}, \boldsymbol{C}\right)$ and associated integral matrix $A_{M}$. Recall that $A_{M}$ is a presentation matrix for the group $H_{1}(M)$; any presentation matrix of $H_{1}(M)$ obtained in this way will be called a t-presentation matrix of $M$. Recall that $A_{M}$ is proper.

Proof of Theorem 1.4 (cf. [6, Theorem 4.3]). The result is obvious for genus one t-manifold structures with the only exception of $S^{3}$, which the hypothesis $A_{M} \neq(1)$ excludes.

Suppose $M$ has a genus $n$ t-manifold structure, where $n>1$; then (a) follows directly from the properness of $A_{M}$, hence $M$ is closed and $H_{1}(M)$ 
is finite. Since any rank $n$ submatrix of $A_{M}$ is a presentation matrix for $H_{1}(M)$, that $H_{1}(M)$ is nontrivial follows from Lemma 5.1.

Let $\{H, \boldsymbol{D}, \boldsymbol{C}\}$ be a t-manifold structure of some t-manifold $M$. Since, by Theorem 1.4, the manifold $M$ is closed, the set $H^{\prime}=\overline{M \backslash H}$ is a handlebody and hence the pair $\left(H, H^{\prime}\right)$ is a Heegaard splitting of $M$. That the curves in $C$ intersect the meridians of $H$ coherently easily proves the following result:

Corollary 6.1. Let $M$ be a t-manifold with a $k \times n$ t-presentation matrix $A_{M}$. If any of the entries of $A_{M}$ is \pm 1 , then the Heegaard genus of $M$ is strictly less than $n$.

Some more information on the topology of a given t-manifold can be obtained if a suitable t-manifold structure is at hand. For example, many of the t-manifold structures in Section 3 arise from Heegaard diagrams of minimal genus. We will see how to partially generalize this situation to higher genus t-manifold structures in the cases when the t-presentation matrix is square, where some of the results of Section 5 on 0-girths of proper matrices can be exploited to give topological information on the t-manifold.

Let $M$ be a t-manifold with t-manifold structure $\boldsymbol{S}=\left\{H_{n}, \boldsymbol{D}, \boldsymbol{C}\right\}$ and t-presentation matrix $A_{M}$. Then $\boldsymbol{C}$ has at most $3 n-3$ components and represents all $n$-types. Now, the $2^{n}$ different $n$-types come in pairs $\varepsilon,-\varepsilon$, and clearly every $k \times n$ proper matrix with no zero entries must be t-equivalent to the matrix whose rows are the vectors $\varepsilon$ just described; in particular, $k=2^{n-1}$. We combine these two simple observations with the main theorem of $[\mathbf{3}]$ to prove the following result:

Lemma 6.2. Let $M$ be a t-manifold with a genus $n$-manifold structure $\left\{H_{n}, \boldsymbol{D}, \boldsymbol{C}\right\}$ and associated Heegaard splitting $\left(H_{n}, H_{n}^{\prime}\right)$. If $n \geq 5$ then the splitting $\left(H_{n}, H_{n}^{\prime}\right)$ is not strongly irreducible. In particular, either $\left(H_{n}, H_{n}^{\prime}\right)$ is reducible or $M$ contains an incompressible closed surface of positive genus.

Proof. Since $3 n-3<2^{n-1}$ for $n \geq 5$, our previous observations imply that $A_{M}$ must have at least one zero entry. This implies that for some circle $c \in C$ and disk $D \in D, c \cap D=\emptyset$, hence the splitting is not strongly irreducible and the last part of the lemma follows from [3, Theorem 3.1].

The conclusion of the previous lemma can be considerably strengthened in the special case when the t-manifold structure gives a Heegaard splitting of minimal genus. This is the content Theorem 1.6, whose proof we proceed to give. In the process, we will follow the notation of $[3, \S 3]$ closely.

Proof of Theorem 1.6. Let the t-manifold structure of $M$ be given by the data $\boldsymbol{S}=\left\{H_{n}, \boldsymbol{D}=\left\{D_{1}, \ldots, D_{n}\right\}, \boldsymbol{C}=\left\{c_{1}, \ldots, c_{n}\right\}\right\}$ and let $H_{n}^{\prime}$ be the handlebody in $M$ complementary to $H_{n}$. We think of the circles in $\boldsymbol{C}$ as the meridian circles of $H_{n}^{\prime}$, which bound disjoint properly embedded disks 
$\boldsymbol{D}^{\prime}=\left\{D_{1}^{\prime}, \ldots, D_{n}^{\prime}\right\}$ in $H_{n}^{\prime}$. Since the Heegaard genus of $M$ is $n, M$ will be reducible once the Heegaard splitting $\left(H_{n}, H_{n}^{\prime}\right)$ is reducible.

Recall from Theorem 1.5 that the 0 -girth of $A_{M}$ is at most $n-1$. If the 0 -girth of $A_{M}$ is $n-1$, then there are $k$ meridian disks of $H_{n}$, say $\boldsymbol{D}^{*}=\left\{D_{1}, \ldots, \partial D_{k}\right\}$, and $l$ meridian disks of $H_{n}^{\prime}$, say $\boldsymbol{C}^{*}=\left\{D_{1}^{\prime}, \ldots, D_{l}^{\prime}\right\}$, which are pairwise disjoint, such that $k, l>0$ and $k+l=n-1$. Observe that the circles $\partial \boldsymbol{C}^{*} \cup \partial \boldsymbol{D}^{*}$ are homologically independent in the Heegaard surface $F=\partial H_{n}$; hence $T=\sigma\left(F ; \partial \boldsymbol{C}^{*} \cup \partial \boldsymbol{D}^{*}\right)$ is a torus and so $c\left(\partial \boldsymbol{C}^{*} \cup \partial \boldsymbol{D}^{*}\right)=$ $2 n-2$.

If the torus $T$ is incompressible in $M$ we are done, so we may assume $T$ compresses. By the proof of [3, Theorem 3.1], it follows that either the Heegaard splitting $\left(H_{n}, H_{n}^{\prime}\right)$ is reducible or there are nonempty collections $\boldsymbol{E}, \boldsymbol{E}^{\prime}$ of disjoint, properly embedded disks in $H_{n}, H_{n}^{\prime}$, respectively, such that

$$
\partial \boldsymbol{E} \cap \partial \boldsymbol{E}^{\prime}=\emptyset \text { and } c\left(\partial \boldsymbol{E} \cup \partial \boldsymbol{E}^{\prime}\right)>c(\partial \boldsymbol{E}), c\left(\partial \boldsymbol{E}^{\prime}\right),
$$

with $c\left(\partial \boldsymbol{E} \cup \partial \boldsymbol{E}^{\prime}\right)>c\left(\partial \boldsymbol{C}^{*} \cup \partial \boldsymbol{D}^{*}\right)=2 n-2$. But, for any 1-submanifold $\alpha$ of $F, c(\alpha) \leq 2 n-1$, with equality holding only if $\sigma(F ; \alpha)$ consists of 2 spheres. Hence $\sigma\left(F ; \partial \boldsymbol{E} \cup \partial \boldsymbol{E}^{\prime}\right)$ is a union of 2 -spheres and, by the proof of $\left[\mathbf{3}\right.$, Theorem 3.1], the Heegaard splitting $\left(H_{n}, H_{n}^{\prime}\right)$ again must be reducible. The last part of the theorem follows immediately from Lemma 5.10.

Proof of Theorem 1.7. We assume here that $X(s)$ has no lens space connected summand; the case when $X(s)$ has no t-manifold summand is similar (cf. [15, Theorem 1.3]). By [6, Theorem 4.3], $X(r)$ always has a t-manifold summand.

Suppose that $X(r)$ has two prime connected summands $M_{1}$ and $M_{2}$, neither of which is part of a t-manifold summand. Let $\widehat{P}$ be a 2 -sphere in $X(r)$ which 'separates' $M_{1}$ from $M_{2}$, and such that $P=\widehat{P} \cap X$ is properly embedded in $X$ with $|\partial P|$ smallest subject to these constraints; then $P$ is essential in $X$ with boundary slope $r$. Let $Q$ be an essential planar surface in $X$ with boundary slope $s$; we may assume that $P$ and $Q$ intersect transversely and that any circle component of $P \cap Q$ is nontrivial in both $P$ and $Q$. Since $X(s)$ has no lens space connected summand, the graph $G_{Q}=P \cap Q \subset Q$ represents all types by [8]. Let $\Sigma$ be a generalized Scharlemann cycle of $G_{Q}$; attaching $\Sigma$ to $\widehat{P}$ (see the Introduction) produces a 2 -sphere $\widehat{P^{\prime}}$ in $X(r)$ which cobounds a t-manifold with $\widehat{P}$ and satisfies $\left|\partial P^{\prime}\right|<|\partial P|$. Since neither manifold $M_{1}$ nor $M_{2}$ is part of a t-manifold summand of $X(r)$, it follows that $\widehat{P^{\prime}}$ 'separates' $M_{1}$ from $M_{2}$ in $X(r)$, contradicting the minimality of $|\partial P|$. The theorem follows.

We end this section with two examples of proper matrices and a couple of open questions. 


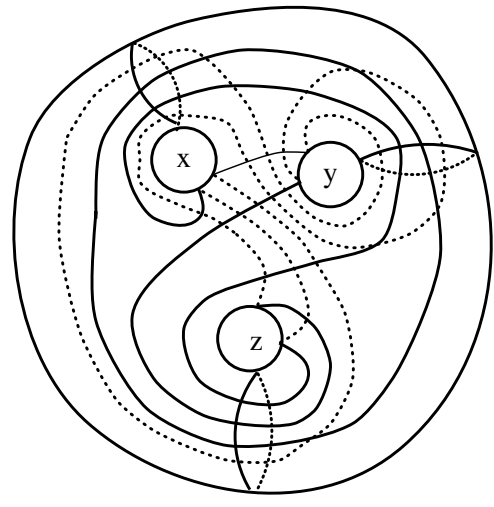

(a)

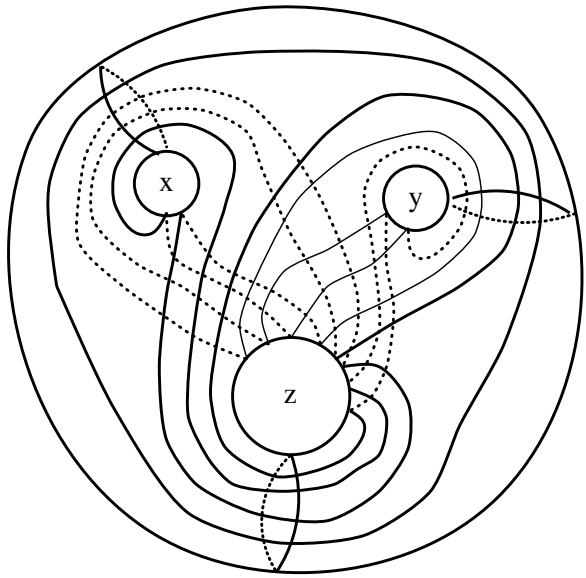

(b)

Figure 12. Two realizations of the matrix $A$.

Consider the matrix

$$
A=\left(\begin{array}{rrr}
1 & 1 & 1 \\
1 & 1 & -1 \\
1 & -1 & 1 \\
1 & -1 & -1
\end{array}\right) .
$$

Figure 12 shows two t-manifold structures with t-presentation matrix $A$; in the case of Figure 12(a) and (b) the t-manifold has fundamental group isomorphic to $\mathbb{Z} / 2 \mathbb{Z} * \mathbb{Z} / 2 \mathbb{Z}$ and $Q_{8}$, respectively, where $Q_{8}=\langle u, v| u^{2}=$ $\left.(u v)^{2}=v^{2}\right\rangle$ is the classical quaternion group; hence the manifold in Figure 12(a) is homeomorphic to $\boldsymbol{P}^{3} \# \boldsymbol{P}^{3}$ (see Corollary 6.1). In particular, there exist reducible $t$-manifolds.

It can be proved that the fundamental group of any t-manifold with tpresentation matrix $A$ is isomorphic to one of the two groups above [14].

Another interesting example involves the matrix

$$
F=\left(\begin{array}{rrrr}
1 & 1 & 1 & 0 \\
1 & -1 & 0 & 1 \\
1 & 0 & -1 & -1 \\
0 & 1 & -1 & 1
\end{array}\right)
$$

It is not hard to see that any proper $4 \times 4$ matrix with 0 -girth at most 2 is t-equivalent to $F$. However, $F$ is not a t-presentation matrix for any t-manifold $M$ with t-manifold structure $\boldsymbol{S}=\left\{H_{4}, \boldsymbol{D}=\left\{D_{1}, \ldots, D_{4}\right\}, \boldsymbol{C}=\right.$ $\left.\left\{c_{1}, \ldots, c_{4}\right\}\right\}$. To see this, let $x_{1}, \ldots, x_{4}$ represent generators of $\pi_{1}\left(H_{4}\right)$ dual to the $D_{i}$ 's, respectively; then $\pi_{1}(M)=\left\langle x_{1}, \ldots, x_{4} \mid c_{1}, \ldots, c_{4}\right\rangle$. Due to the 
coherency of the intersections between the $\partial D_{i}$ 's and the $c_{j}$ 's, there are only a few choices for each word $c_{i}$. For example, we may have that, up to cyclic permutation,

$$
c_{1}=x y z, c_{2}=x y^{-1} w, c_{3}=x z^{-1} w^{-1}, c_{4}=y z^{-1} w,
$$

which would yield

$$
\pi_{1}(M) \approx G=\left\langle x, t \mid x^{3} t^{3}=1, t x t^{-1}=x^{-2}\right\rangle
$$

after substituting $w=x t$. A little more computation shows that all the possible groups $\pi_{1}(M)$ obtained in this way are isomorphic to $G$ [14]; hence in all cases $\pi_{1}(M)$ is nilpotent of order 27 , and $H_{1}(M) \approx \mathbb{Z} / 3 \mathbb{Z} \oplus \mathbb{Z} / 3 \mathbb{Z}$. This contradicts the fact that any finite nilpotent 3 -manifold group is cyclic or has even order [5], which proves our claim.

The above example of the $4 \times 3$ proper matrix $A$ shows that a t-manifold may be reducible; in that particular case, however, we can see that the prime factors of the t-manifold are also t-manifolds (in fact, lens spaces). So we ask:

Question 1. Are the prime factors of any t-manifold also t-manifolds?

A positive answer to this question would improve the conclusion of Theorem 1.7 to say: If $X(s)$ has no lens space connected summands, then at most one of the prime factors of $X(r)$ is not a $t$-manifold.

By Theorem 1.1, no t-manifold with a genus two t-manifold structure is hyperbolic, and Theorem 1.6 provides many more examples of non hyperbolic t-manifolds. So we ask:

Question 2. Are there any hyperbolic t-manifolds?

A negative answer to this last question would imply that t-manifolds are exceptionally rare, as would be the reducible surgeries giving rise to them.

\section{References}

[1] C. Berge, The Theory of Graphs and its Applications, Wiley, London, Methuen, New York, 1964, MR 24 \#A2381, Zbl 0097.38903.

[2] R.A. Brualdi and H.J. Ryser, Combinatorial Matrix Theory, Encyclopedia of Mathematics and its Applications, 39, Cambridge University Press, New York, NY, 1991, MR 93a:05087, Zbl 0746.05002.

[3] A.J. Casson and C.McA. Gordon, Reducing Heegaard splittings, Topology Appl., 27 (1987), 275-283, MR 89c:57020, Zbl 0632.57010.

[4] M. Culler, C.McA. Gordon, J. Luecke and P. Shalen, Dehn surgery on knots, Ann. of Math., 125 (1987), 237-300, MR 88a:57026, Zbl 0633.57006.

[5] B. Evans and L. Moser, Solvable fundamental groups of compact nilpotent 3-manifolds, Trans. Amer. Math. Soc., 168 (1972), 189-210, MR 46 \#897, Zbl 0241.57002. 
[6] C.McA. Gordon, Combinatorial methods in Dehn surgery, Proceedings of the Workshop of 'Knots '96,' The Fifth MSJ International Research Institute in Knot Theory (Waseda University, Tokyo, Japan), 1997, 263-290, MR 98m:57020, Zbl 0940.57022.

[7] C.McA. Gordon and R.A. Litherland, Incompressible planar surfaces in 3-manifolds, Topology Appl., 18 (1984), 121-144, MR 86e:57013, Zbl 0554.57010.

[8] C.McA. Gordon and J. Luecke, Knots are determined by their complements, J. Amer. Math. Soc., 2 (1989), 371-415, MR 90a:57006a, Zbl 0678.57005.

[9] _ Reducible manifolds and Dehn surgery, Topology, 35 (1996), 385-409, MR 97b:57013, Zbl 0859.57016.

[10] P. Hall, On representatives of subsets, J. London Math. Soc., 10 (1935), 26-30, Zbl 0010.34503.

[11] P.R. Halmos and H.E. Vaughan, The marriage problem, Amer. J. Math., 72 (1950), 214-215, MR 11,423h, Zbl 0034.29601.

[12] M. Jankins and W.D. Neumann, Lectures on Seifert Manifolds, Brandeis Lecture Notes, 2, Brandeis University Press, Chicago, Il, 1983, MR 85j:57015.

[13] W. Parry, All types implies torsion, Proc. Amer. Math. Soc., 110 (1990), 871-875, MR 91c:20079, Zbl 0765.20027.

[14] L.G. Valdez Sánchez, On Manifolds Obtained from Generalized Scharlemann Cycles, Ph.D. Thesis, UC Berkeley, 1995.

[15] _ Dehn fillings of 3-manifolds and non-persistent tori, Topology Appl., 98(1-3) (1999), 355-370, MR 2000g:57034, Zbl 0935.57024.

Received June 1, 1999.

Department of Mathematical Sciences

University of Texas at El Paso

El PASO, TX 79968

E-mail address: valdez@math.utep.edu 